\title{
Jet Noise Prediction Comparisons with Scale Model Tests and Learjet Flyover Data
}

\author{
Brenda Henderson, Dennis Huff, Jeff Berton \\ NASA Glenn Research Center, Cleveland, Ohio 44135
}

\begin{abstract}
Recent interest in commercial supersonic flight has highlighted the need to accurately predict Effective Perceived Noise Levels (EPNL) for aircraft and, since the dominant noise source at takeoff will likely be jet noise, specifically jet noise contributions. The current study compares predictions from historical jet-noise models within NASA's Aircraft Noise Prediction Program and scale-model data to measurements made in a Learjet 25 flight test. The noise levels from the predictions and scale-model data were below those for the flight data by $2.5-3.5,1-2$, and 3 5 EPNdB for the SAE model, the Stone Jet model, and the scale-model data, respectively. Tones and broadband haystacks were identified in the flight spectra that are not associated with jet noise which increased the flight EPNL by at least 0.5 EPNdB over that computed from spectra with the tones and haystacks removed. The study highlights the need for accurate exhaust temperature measurements, aircraft flight position information, and averaging data across a line of microphones in flight tests. For example, a $100^{\circ} \mathrm{F}$ to $200^{\circ} \mathrm{F}$ difference in jet exhaust temperature is enough to explain the differences between flight, model scale, and prediction comparisons.
\end{abstract}

\section{Introduction}

A renewal of interest in commercial supersonic flight has led to an on-going effort by the International Civil Aviation Organization (ICAO) Working Group 1/Supersonic Task Group (WG1/SSTG) within the Landing and Take-off (LTO) subgroup to develop noise standards and recommended practices (SARPs) for the airport environment. In support of this effort, NASA is conducting studies to predict noise for representative supersonic aircraft and a range of take-off and landing procedures. The studies have shown jet-noise to be the dominant noise source at the lateral and flyover locations and have highlighted the need to accurately predict jet noise for relevant exhausts. For the types of studies included in the effort, three approaches are available for jet-noise prediction: employing empirical models, scaling data acquired in wind tunnels, and using scaled engine test-stand data from similar engines with an added forward flight correction. The current study investigates the first two prediction approaches and compares the results to data previously acquired in a Lear 25 flight test. The flight data are compared to scale model data acquired in NASA's Aero-Acoustic Propulsion Laboratory and predictions using empirical models currently available in NASA's Aircraft Noise Prediction Program (ANOPP) ${ }^{1}$. The Learjet dataset was selected for comparison as the dominant noise source for the aircraft is expected to be jet noise. The Learjet dataset was used previously to quantify the noise reduction associated with the introduction of chevrons. However, the initial study was not focused on jet-noise prediction and, therefore, did not include an accurate scale-model of the Lear 25 nozzle system or comparisons with empirical models².

Plans for near-term entry-into-service supersonic aircraft appear to be focused on business jets ${ }^{3,4}$ with 2 or 3 engines and subsonic overland flight. The jet flows will likely be fully mixed single streams exhausting from turbofan engines for performance and limited to high subsonic to low supersonic speeds to meet airport noise requirements. While the Lear 25 turbojet engines have pseudo secondary streams as a result of NACA scoops and vents near the front of the nacelle that draw in cooling air, the secondary streams are likely at very low speeds relative to the primary exhaust streams. The resulting acoustic spectra from scale model tests will be shown to be similar to those from a single stream jet over the range of frequencies of interest in this study. Additionally, the flight dataset includes points with high subsonic and low supersonic exhaust speeds making portions of the data relevant to future supersonic business jets.

The NASA Aircraft Noise Prediction Program contains a number of jet-noise source models including those based on SAE $876^{5}$, modifications to SAE $876^{6}$, and work performed by Stone ${ }^{7,8}$. The SAE models were developed in the 1970s and were based on data available at the time. Comparisons with flight data ${ }^{10,11}$ have shown the measured levels can be higher than those predicted with the differences being attributed to contamination of the flight data by other noise sources and by installation effects ${ }^{10}$. More recently, the SAE method was found to overpredict levels for engines in the 3000 to $6000 \mathrm{lb}$ thrust class when compared to data collected from an engine- 
stand test ${ }^{6}$. The engine-stand data were obtained from a turbo-fan engine with a lobed mixer. The lower levels in the engine-stand test resulted in a modified SAE model within ANOPP. The original jet-noise model developed by Stone $^{7}$ for single stream jets was based on data available in the 1970s (referred to as Stone 1 here) and is available in ANOPP. Stone later updated the model using data acquired more recently in scale-model experiments and extended the model to predict noise for dual-stream jets ${ }^{8}$. The newer Stone model, referred to as Stone 2 here, is also available in ANOPP. It will be shown that the SAE, modified SAE, Stone 1, and Stone 2 models produce different source distributions for the jet exhausts explored here. Not included in this study are models developed more recently ${ }^{12,13,14}$ as these intent of the investigation is to apply models currently available within ANOPP.

The quantity of interest is the effective perceived noise level (EPNL). However, in this study, spectral shapes and levels over the observation angles of interest (those angles contributing to the EPNL calculation) will be investigated in addition to the EPNL. The intent is to fully understand the predictions contributing to the EPNL and how those predictions compare to scale model and flight data. Only mixing noise will be considered in this study. Models for broadband shock noise will not be investigated.

\section{Experiments}

\section{A. Flight Tests}

A flight test was conducted in March 2001 at the Estrella Sailport (located south of Phoenix, Arizona) using a Learjet 25 operated by NASA ${ }^{2}$. The primary purpose of the test was to verify noise reduction benefits of chevron nozzles relative to the baseline nozzles for General Electric CJ610 turbojet engines (see Fig. 1). Flyover tests were done over three days, the first of which was for the baseline nozzles. During 500-ft. constant altitude, constant speed flyovers (see Fig. 2), the left engine was set at the desired power condition while the right engine was throttled back to idle. Only the baseline data are presented here.

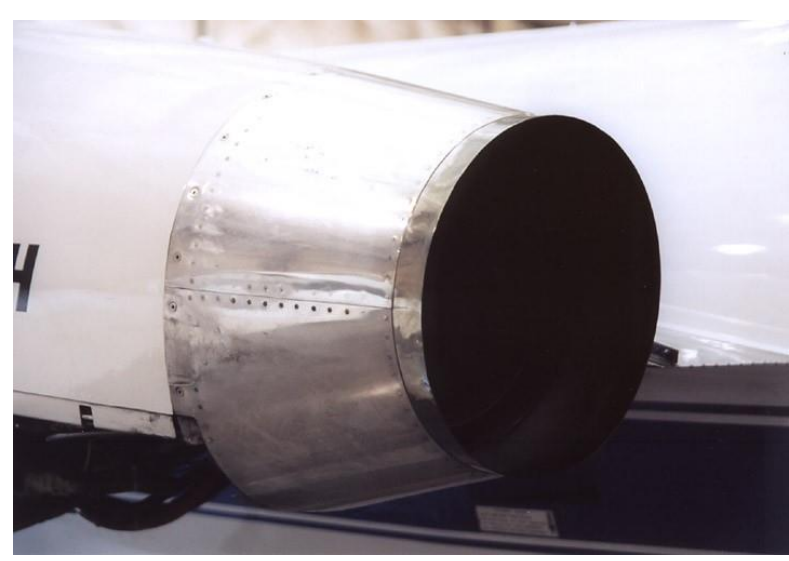

Figure 1. The baseline nozzle mounted on

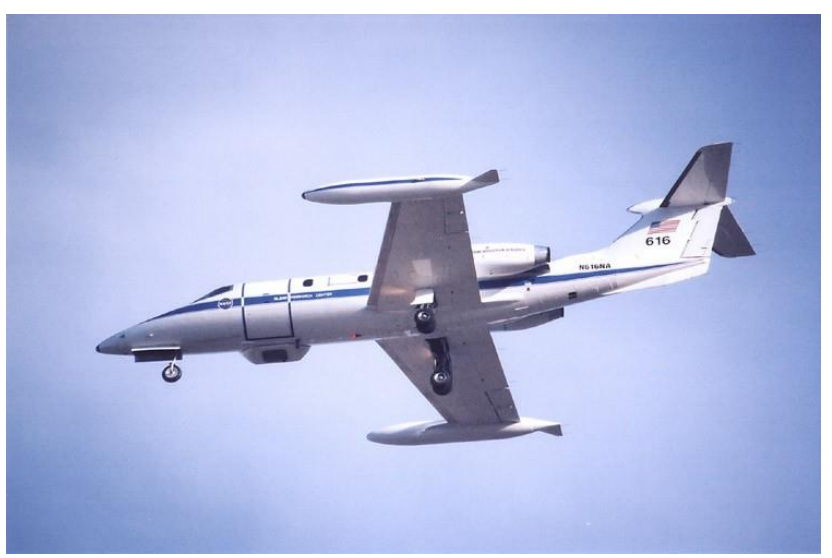

Figure 2. The flyover of the Learjet 25 with the zles.

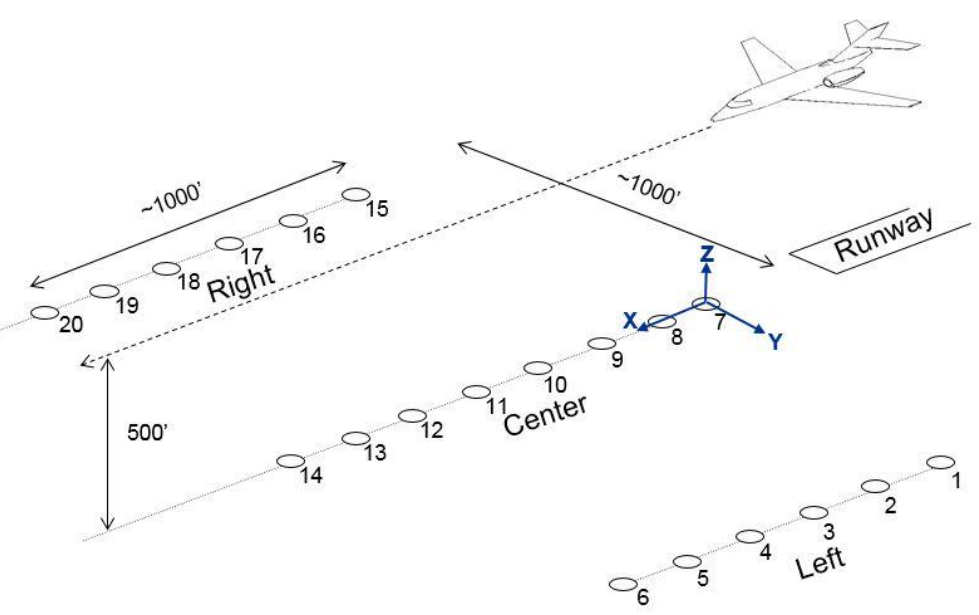

Figure 3. The ground microphone array and coordinate system. 
Table 1 Microphone Locations

\begin{tabular}{|c|c|c|c|}
\hline & \multicolumn{2}{|c|}{ Location of Microphone Relative to Microphone 7 (feet) } \\
\hline Microphone & $\mathbf{X}$ & $\mathbf{Y}$ & $\mathbf{Z}$ \\
\hline 1 & 150.03 & 1000 & -6.83 \\
\hline 2 & 299.99 & 1000 & 0.36 \\
\hline 3 & 449.89 & 1000 & 0.69 \\
\hline 4 & 606.13 & 1000 & 0.62 \\
\hline 5 & 750.08 & 1000 & 0.98 \\
\hline 6 & 900.17 & 1000 & 1.17 \\
\hline 7 & 0 & 0 & 0 \\
\hline 8 & 150.03 & 0 & 0.48 \\
\hline 9 & 300.13 & 0 & 2.03 \\
\hline 10 & 450.65 & 0 & 2.08 \\
\hline 11 & 599.96 & 0 & 2.09 \\
\hline 12 & 750.3 & 0 & 2.27 \\
\hline 13 & 912.71 & 0 & 2.61 \\
\hline 14 & 1050 & 0 & 2.64 \\
\hline 15 & 150.03 & -1000 & 3.3 \\
\hline 16 & 311.3 & -1000 & 1.9 \\
\hline 17 & 449.91 & -1000 & 3.38 \\
\hline 18 & 599.96 & -1000 & 3.07 \\
\hline 19 & 750.05 & -1000 & 3.59 \\
\hline 20 & 900.15 & -1000 & 3.25 \\
\hline
\end{tabular}

Table 2 Flight Test Matrix for Baseline Nozzles

\begin{tabular}{|c|c|c|c|c|c|c|}
\hline $\begin{array}{c}\text { Engine } \\
\text { Pressure } \\
\text { Ratio } \\
\text { (EPR) } \\
\end{array}$ & $\begin{array}{c}\text { Run } \\
\text { Number }\end{array}$ & $\begin{array}{l}\text { Approximate } \\
\text { Jet } \\
\text { Temperature } \\
(\mathbf{R}) \\
\end{array}$ & $\begin{array}{c}\text { Landing } \\
\text { Gear }\end{array}$ & $\begin{array}{c}\text { Ground } \\
\text { Speed } \\
\text { (knots) }\end{array}$ & \begin{tabular}{|c|}
$\begin{array}{c}\text { Temperature } \\
\text { at } \\
\text { Microphone 7 } \\
\text { (F) }\end{array}$ \\
\end{tabular} & $\begin{array}{c}\text { Relative } \\
\text { Humidity } \\
(\%)\end{array}$ \\
\hline \multirow{5}{*}{1.6} & 136 & 1180 & Up & 202.7 & 67.9 & 46.6 \\
\hline & 137 & 1180 & Up & 206.6 & 67.7 & 47.1 \\
\hline & 138 & 1180 & Up & 205.3 & 67.4 & 47.5 \\
\hline & 139 & 1180 & Up & 211.1 & 67.9 & 46.6 \\
\hline & 140 & 1180 & Up & 205.8 & 68.1 & 45.9 \\
\hline \multirow{6}{*}{1.8} & 123 & 1257 & Down & 166.7 & 61.2 & 48 \\
\hline & 124 & 1257 & Down & 157.5 & 60.5 & 48 \\
\hline & 125 & 1257 & Down & 165.8 & 61.6 & 48 \\
\hline & 133 & 1257 & Down & 156.2 & 66.3 & 47.6 \\
\hline & 134 & 1257 & Down & 154.3 & 67.3 & 47.7 \\
\hline & 135 & 1257 & Down & 166.6 & 67.3 & 48.6 \\
\hline \multirow{5}{*}{2} & 121 & 1374 & Down & 187.7 & 61.5 & 48 \\
\hline & 122 & 1374 & Down & 188.6 & 61.2 & 48 \\
\hline & 128 & 1374 & Down & 177.7 & 64.5 & 49.6 \\
\hline & 129 & 1374 & Down & 175.6 & 65.1 & 49.5 \\
\hline & 130 & 1374 & Down & 174.7 & 65 & 50 \\
\hline
\end{tabular}
EPRs, the EGT of the right engine was $54^{\circ} \mathrm{F}$ to $72^{\circ} \mathrm{F}$ below that of the left engine. The approximate jet temperatures
NASA Langley recorded the acoustic data during the flyovers using ground microphones. The microphones were arranged in three lines along the flight direction (left, right and center of the flyover path). Figure 3 is a schematic showing the microphone numbering system and locations relative to the flight path. Table 1 shows the position of each microphone relative to Microphone 7 , which was the first microphone on the center array directly below the flight path. A $1 \frac{1}{2}$-inch $B \& K$ microphone was placed flat on a steel plate for each microphone position. The terrain was mostly dirt with sparse vegetation (desert). Time pressure histories were digitally recorded with a sample rate of 25,000 samples per second. For each line of microphones, the pressures were time shifted to the lead microphone (1, 7, or 15$)$ and averaged using the procedure described in Ref. 15. The flight tests started at 6:34 am on March 27, 2001 and ended at 9:56 am. The temperature at Microphone 7 ranged from 58.1 to $68.1 \mathrm{~F}$ and the relative humidity was about $48 \%$. The pilot set the engine pressure ratio (EPR) to the desired power and deployed flaps and landing gear as appropriate to control the flight speed. The subset of the data used in the current study is shown in Table 2. The flaps were deployed for all of the runs and the landing gear was deployed for the data acquired at $\mathrm{EPR}=1.8$ and 2.0. Several runs were not used due to loss of signal on the microphones, particularly for the left line of microphones. Note that Runs 121 through 125 use estimates for relative humidity based on the local temperature. Engine Exhaust Gas Temperatures (EGT) were determined in a preflight test conducted in
auges for each EPR. For all 
in Table 2 reflect the preflight EGTs for the left engine. Exhaust Gas Temperatures were not recorded during the acoustic flight tests in March 2001.

Time pressure histories were analyzed in 0.50 second intervals using a Fast Fourier Transform (FFT). The number of samples for the FFT was 4096, giving a $6.1 \mathrm{~Hz}$ bandwidth. A Hamming periodic window was chosen with Welch's power spectral density (PSD) estimate. The FFTs acquired from the various microphones along the flight path were averaged using time shifted pressure histories. The time shift was obtained from the aircraft flight speed. The narrowband spectra were converted to 1/3-octave and PNL and EPNL were computed using the same method employed in ANOPP.

\section{B. Scale-Model Tests}

A 0.31 scale factor model of the Learjet nozzle system was designed using relevant nozzle dimensions obtained from detailed measurements of the nozzle used in the flight tests. The leading-edge region of the Learjet nacelle has the NACA scoop and two vents shown in Fig. 4. The air from the scoop and vents exhausts through an outer secondary stream. Both air streams and the slight offset of the secondary nozzle relative to the core nozzle were replicated in the scale-model shown in Fig. 5. The diameter of the core nozzle is 3.59".

The scale-model experiments were performed in the Aero-Acoustic Propulsion Laboratory (AAPL) at the NASA Glenn Research Center (see Fig. 6). The AAPL is a $66 \mathrm{ft}$ radius geodesic dome treated with acoustic wedges. The AAPL contains the Nozzle Acoustic Test Rig (NATR), which produces a 53 in diameter simulated forward flight stream reaching Mach numbers of 0.35 and contains the High Flow Jet Exit Rig (HFJER), a three-stream jet engine simulator capable of replicating most commercial turbo-fan engine temperatures and pressures ${ }^{16}$.

The core- and simulated flight-stream conditions in the experiments closely replicated those used in the flight test (see Table 1). The nozzle pressure ratio (NPR), the ratio of the core pressure to the ambient pressure, was set to the EPR in Table 1 and the nozzle temperature ratio, the ratio of the ambient temperature to the core temperature, was matched to the temperature ratio computed from the approximate temperature in Table 2 and an average ambient temperature for the flight tests. It was not possible to determine the conditions of the secondary flow in the flight tests so pressure ratios equal to $1.05,1.1$, and 1.2 were used in the scale-model experiments to determine the impact of the secondary flow on the resulting spectra. The temperature ratio of the secondary stream was set equal to 1.25. It is expected that the lowest pressure ratio tested, a pressure ratio that resulted in a flow Mach number equal to that of the flight stream, was more representative of the secondary flow in the flight tests than the higher pressure ratios. Data were acquired for two nozzle azimuthal angles: one representing the orientation of the nozzle for the flyover centerline array and one representing the nozzle orientation for the flyover left sideline array.

Acoustic measurements were made with a 24 microphone far-field array located on a 45 foot constant radius arc covering polar angles between $45^{\circ}$ and $160^{\circ}$, where angles greater than $90^{\circ}$ are in the downstream direction relative to the nozzle exit. All data were corrected for atmospheric absorption ${ }^{17}$ and free-jet shear layer effects ${ }^{18}$. Data were acquired using 1/4" Bruel and Kjaer microphones without grid caps, pointed directly at the nozzle exit. Microphone sensitivity and frequency response were applied to all measurements. The narrowband wind tunnel data are presented as power spectral density in emission coordinates on a one-foot lossless arc. For comparisons with jetnoise prediction models within ANOPP, data are presented as Doppler shifted one-third octave sound pressure levels on a one-foot lossless arc to be consistent with the source model representations within ANOPP. For comparisons with flight data, a scale factor of 3.21 is applied to the data and comparisons are made from the scaled data propagated with the ANOPP propagation module ${ }^{19}$. Perceived Noise Levels (PNLs) and EPNLs are computed with ANOPP. 


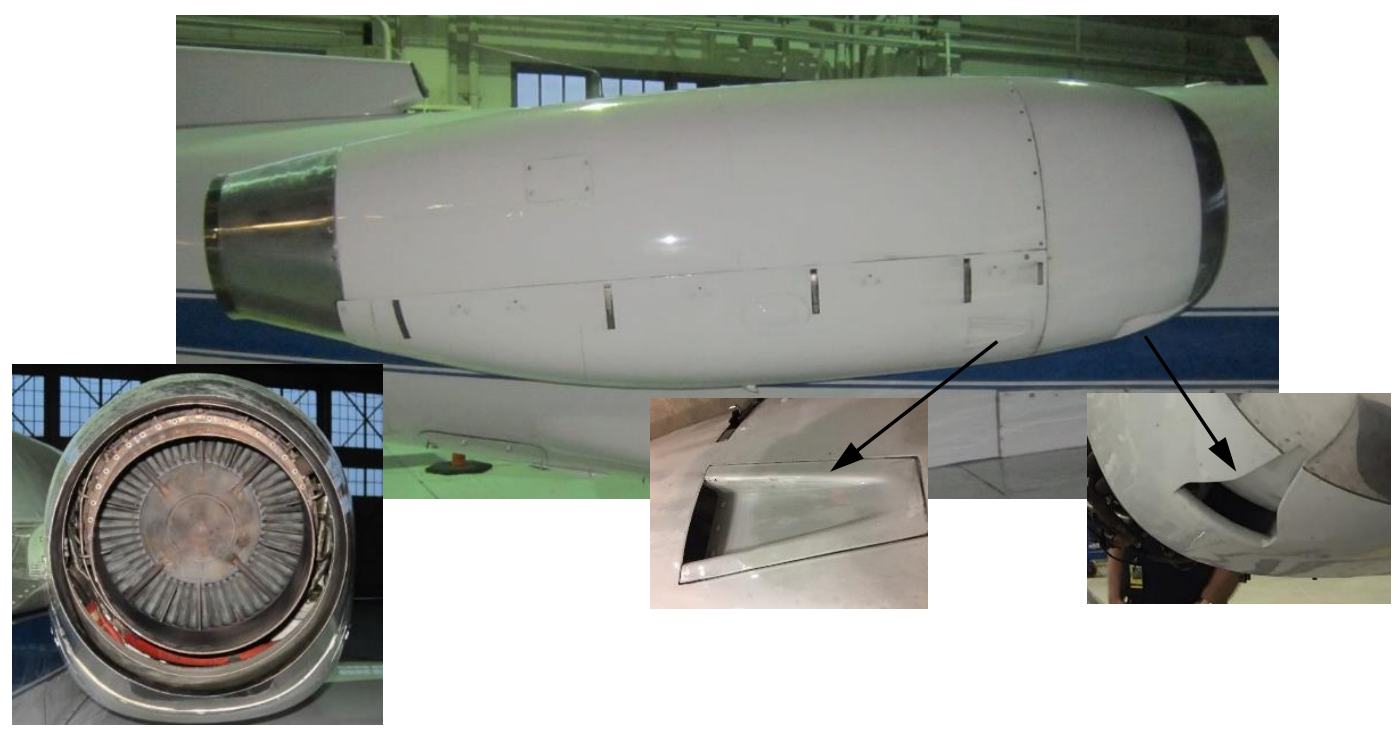

Figure 4. The Learjet nozzle used in the flight.
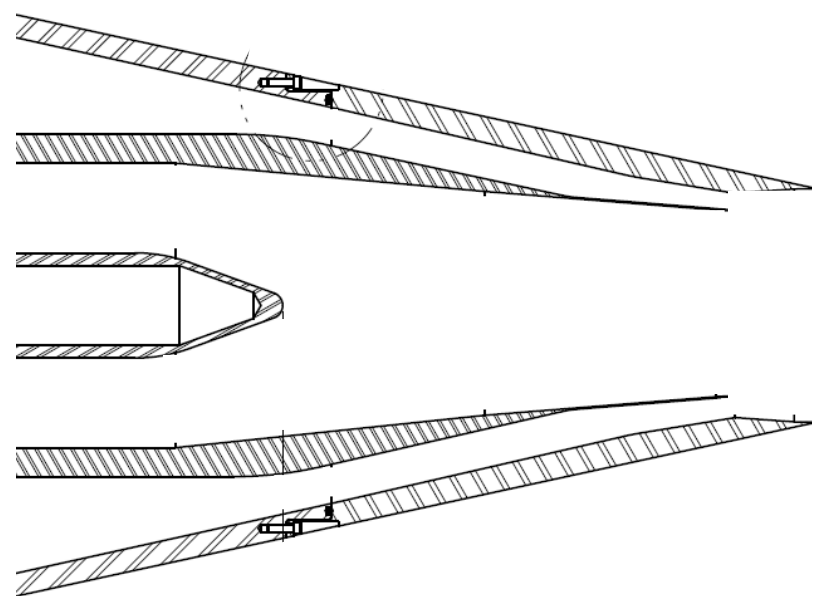

Figure 5. The nozzle system used in the scale model

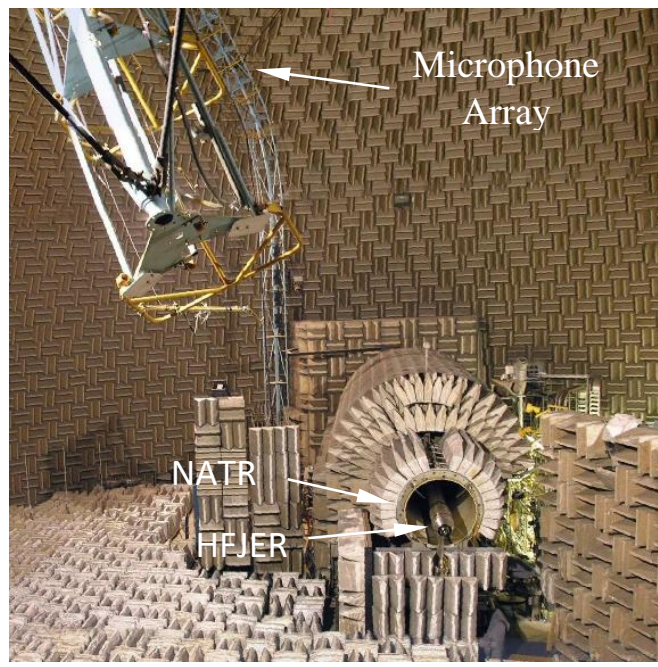

Figure 6. The Aero-Acoustic Propulsion Laboratory (AAPL) at the NASA Glenn Research Center. 


\section{Results}

Calculations of effective perceived noise levels showed that computing EPNL with emission angles between roughly $70^{\circ}$ and $150^{\circ}$ resulted in a value that was within $0.5 \mathrm{EPNdB}$ of the value obtained when using all angles within the required $10 \mathrm{~dB}$ down point on the PNL. Therefore, spectral comparisons with jet-noise source models and flyover data will only be made for angles within this range. Additionally, all EPNL calculations did not use tone corrections as tones were present in the flight data and these tones were not associated with jet noise. The focus of the investigation was jet noise.

\section{A. Comparisons with Scale Model Data}

The narrowband data for an NPR of 2.0 are shown in Fig. 7 for a secondary nozzle pressure ratio $\left(\mathrm{NPR}_{\mathrm{s}}\right)$ of 1.05 . There are no indications of broadband shock noise at any emission angle. As such, comparisons between scale-

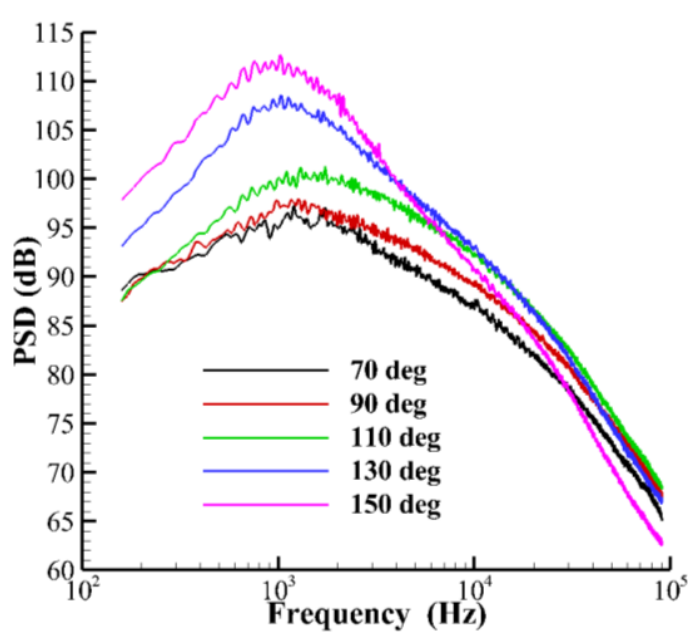

Figure 7. The narrowband spectra acquired at $\mathrm{NPR}=\mathbf{2 . 0}$ and $\mathrm{NPR}_{\mathrm{s}}=\mathbf{1 . 0 5}$.

model data and jet-noise predictions models within ANOPP will only use mixing noise models.

The narrowband spectra acquired at NPR $=1.8$ and $\mathrm{NPR}_{\mathrm{s}}=$ 1.05 are shown in Fig. 8 for six different acquisitions and two different azimuthal angles. The data repeatability is quite good with the exception of tones that occurred for one installation at small angles to the inlet jet axis [see Fig. 8 (a)]. These tones appear to be associated with a particular installation of the nozzle system. The azimuthal nozzle clocking angle did not impact the acoustic radiation likely due to the fact that there was little secondary-stream flow and the offset was reasonably small. The EPNLs computed with spectra containing tones were the same as those computed with tone-free spectra. The results in Fig. 8 are representative of those obtained at other jet operating conditions.

The narrowband spectra for NPR $=1.6$ and 1.8 with $1.05 \leq$ $\mathrm{NPR}_{\mathrm{s}} \leq 1.20$ are shown in Figs. 9 and 10, respectively.

Increasing NPRs from 1.05 to 1.10 slightly decreases high frequency acoustic radiation at all emission angles for NPR = 1.60 and slightly decreases high frequency acoustic radiation in the aft quadrant for NPR $=1.80$. Increasing $\mathrm{NPR}_{\mathrm{s}}$ from 1.10 to 1.20 increases acoustic radiation at low frequencies for all observation angles and both core nozzle pressure ratios. Results for NPR $=2.0$ were similar to those for NPR $=1.60$.

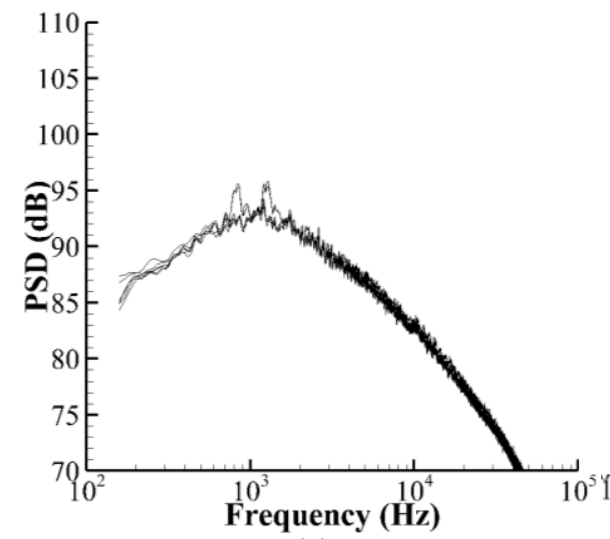

(a)

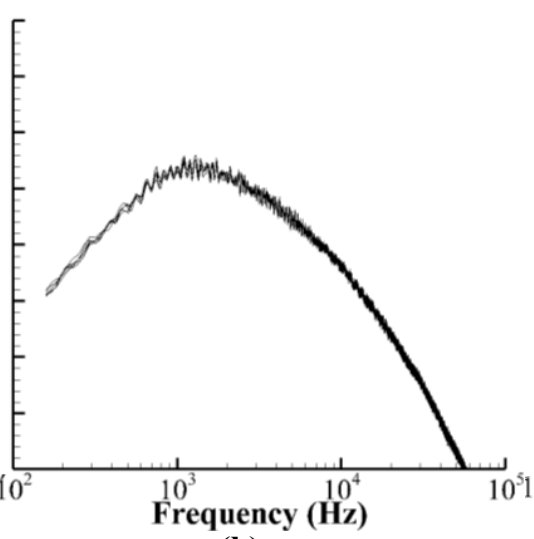

(b)

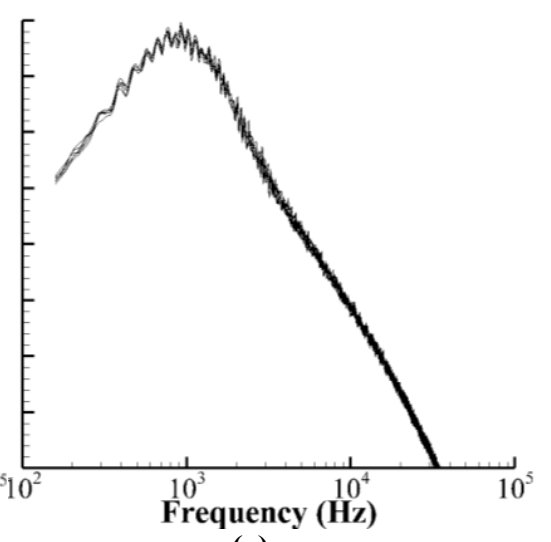

(c)

Figure 8. The narrowband spectra acquired at $N P R=1.8$ and $N P R_{s}=1.05$ for emission angles equal to (a) $7^{\circ}$, (b) $110^{\circ}$, and (c) $150^{\circ}$. Data from six separate acquisitions are included in the plots. The data were acquired at two different azimuthal angles. 


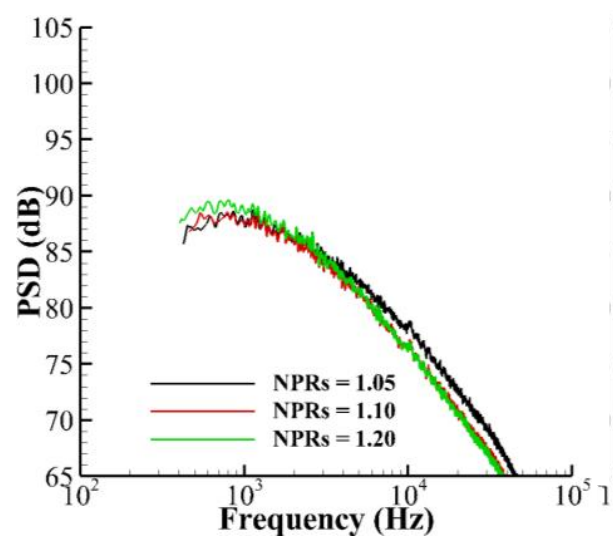

(a)

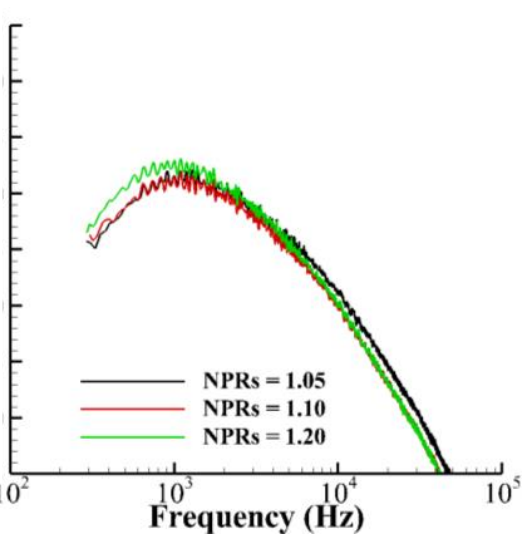

(b)

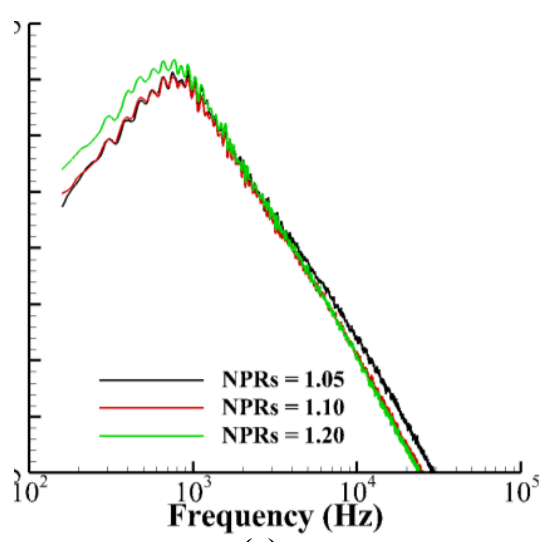

(c)

Figure 9. The narrowband spectra acquired at $N P R=1.6$ and $N P R_{s}=1.05,1.10$ and 1.20 for emission angles equal to (a) $70^{\circ}$, (b) $110^{\circ}$, and (c) $150^{\circ}$.

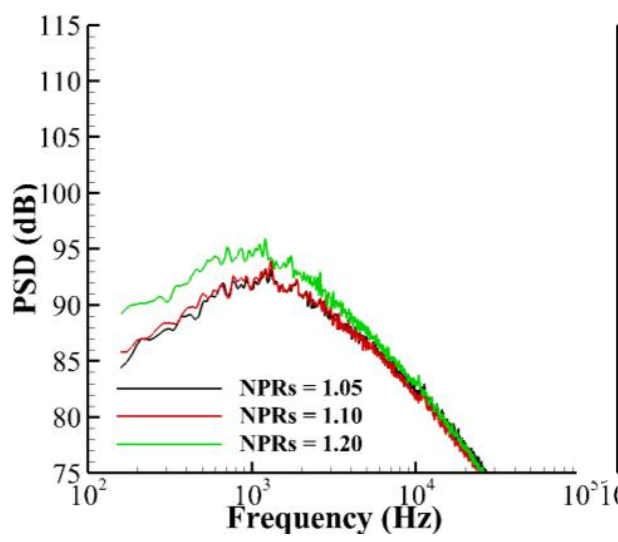

(a)



(b)

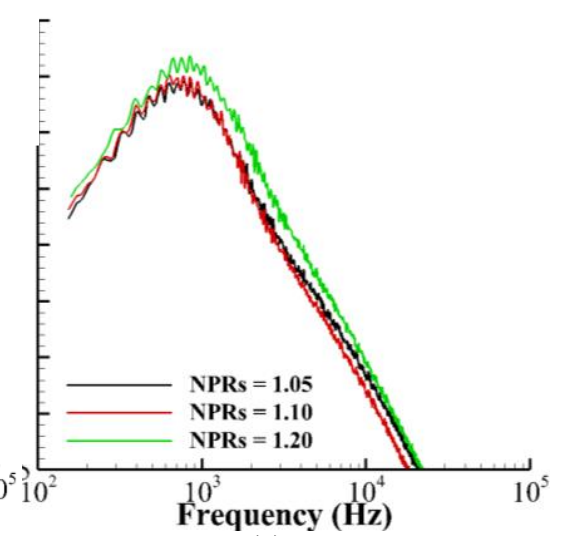

(c)

Figure 10. The narrowband spectra acquired at $N P R=1.8$ and $N P R_{s}=1.05,1.10$ and 1.20 for emission angles equal to (a) $70^{\circ}$, (b) $110^{\circ}$, and (c) $150^{\circ}$.

The free-jet shear layer correction ${ }^{18}$ can be used with the sound source assumed to be at the nozzle exit or distributed. The distributed source in Ref. 18 is based on data acquired from jets tested statically. For the implementation used here, the source distribution in Ref. 18 has been slightly modified by an assumed potential core length. The same potential core length was used for all operating conditions. Comparisons were made between the data corrected for the sources at the nozzle exit (referred to as NATR Exit), distributed sources using the assumed potential core length (referred to as NATR Medium), distributed sources using a potential core length twice that for NATR Medium (referred to as NATR Long), and distributed sources with a potential core length equal to one-half that of NATR Medium (referred to as NATR Short). The results are shown in Fig. 11 for the NPR = 1.8 and NPR $_{s}=$ 1.05. The data have been averaged. For small and nearly broadside angles to the jet [see Figs. 11 (a) and (b)] the peak levels for spectra with the sources assumed at the nozzle exit are lower than those for the distributed sources. At small angles to the jet [see Fig. 11 (a)] mid-frequency levels are slightly impacted by the selection of potential core length. In the peak jet-noise direction [Fig. 11 (c)], the peak level for NATR Exit is higher than those for the data corrected with distributed source. The potential core length only impacts acoustic levels at high frequencies. 


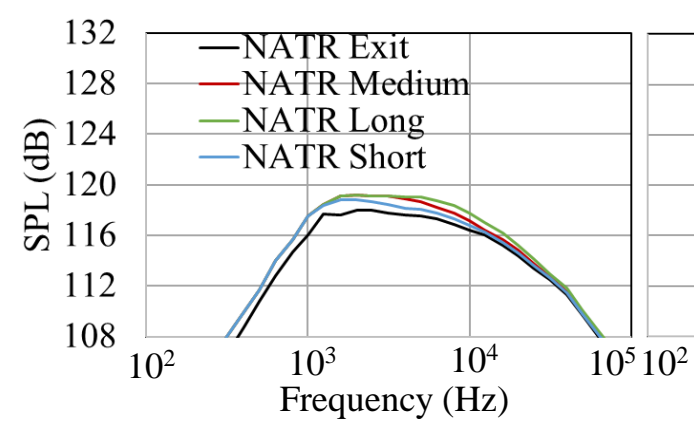

(a)

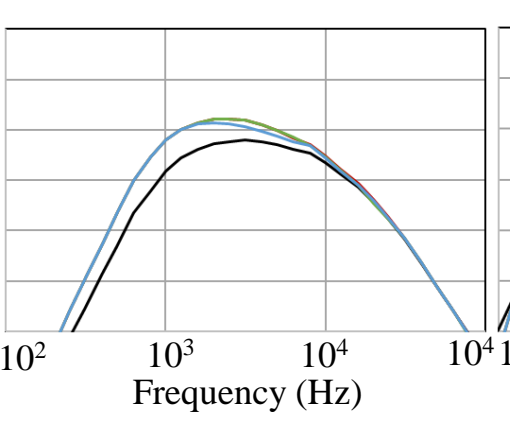

(b)

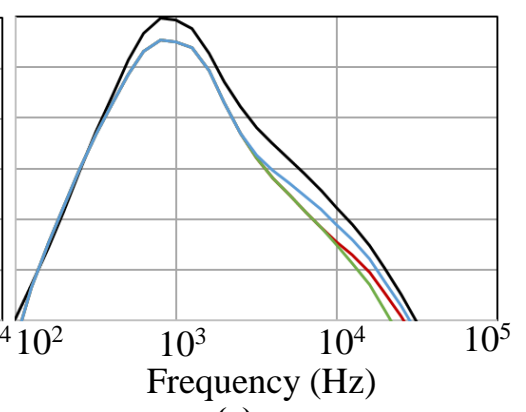

(c)

Figure 11. The one-third octave spectra acquired at $N P R=1.8$ and $N P R_{s}=1.05$ for emission angles equal to (a) $70^{\circ}$, (b) $110^{\circ}$, and (c) $150^{\circ}$. The different source distribution assumptions assumed for the shear laver correction are indicated in the legend.

A comparison between the measured data and SAE, Stone 1, and Stone 2 predictions are shown in Fig. 12. For Stone 2, only the intermediate and large scale sources have been selected in ANOPP. Inclusion of Stone 2 small scale sources results in significant overpredictions of high frequency levels relative to the measured values. The single stream option within Stone 2 was selected for the predictions as the secondary stream had a very low pressure and flow rate in the experiments. The modified SAE predictions are not shown as the levels for modified SAE are 1 $\mathrm{dB}$ below those for SAE at all angles and frequencies. Only the data for NATR Exit and NATR Medium have been included in the Figure. For emission angles in the forward arc [Fig. 12 (a)], Stone 1 and Stone 2 spectra are close to the NATR Exit and NATR Medium spectra, respectively. The highest spectral levels occur for the SAE prediction. For angles near the broadside of the jet [see Fig. 12 (b)], Stone 1 and NATR Exit produce similar spectra while spectra from SAE and NATR Medium are quite similar. Near the peak jet-noise angle [Fig. 12 (c)], peak levels for Stone 1, SAE, and NATR Exit are similar and the spectral shapes of SAE and NATR Exit are similar. The slight differences in the SAE and NATR Exit spectra near $3100 \mathrm{~Hz}$ may be due to the secondary stream in the nozzle system used in the experiments. For emission angles in the aft quadrant, Stone 2 produces the highest peak levels with a frequency that is slightly higher than those from the measurements and other predictions. A comparison between the predicted and measured spectra for NPR $=1.6$ in Fig. 13 show similar trends to those for NPR $=1.8$ although the difference in the peak levels relative to the measure data for SAE in the forward quadrant and for Stone 2 in the peak jet-noise direction are slightly great than those at NPR $=1.8$. The comparisons between measured and predicted spectra at NPR $=2.0$ in Fig. 14 once again shows similar trends to those for NPR $=1.8$ although the comparisons between Stone 1 and measured data are not as good in the forward arc and the peak jet-noise direction as those for NPR $=1.8$.

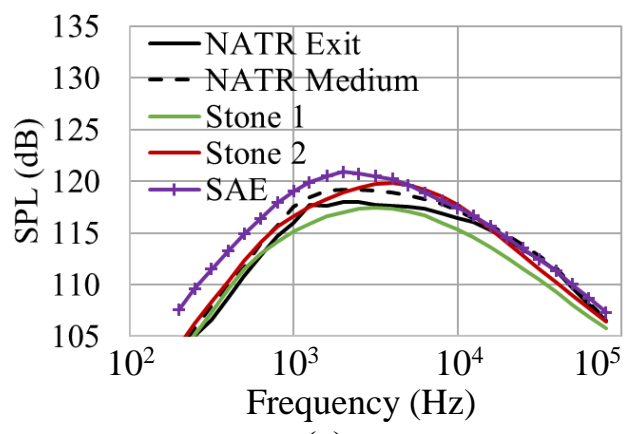

(a)

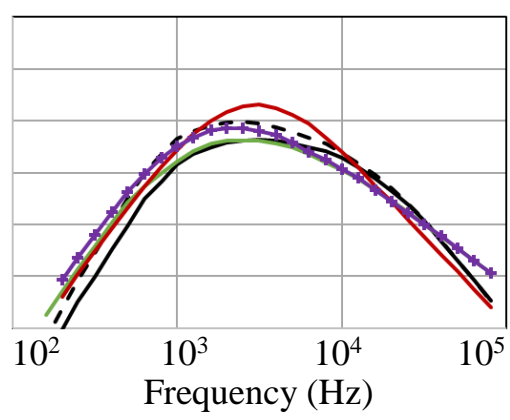

(b)

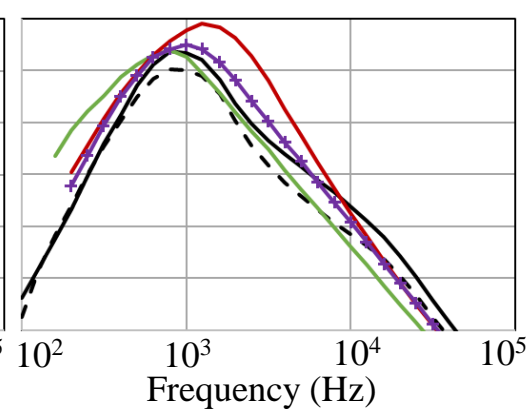

(c)

Figure 12. The one-third octave spectra from ANOPP predictions and measurements at NPR $=1.8$ for emission angles equal to (a) $70^{\circ}$, (b) $110^{\circ}$, and (c) $150^{\circ}$. The NATR spectra are from averaged data and acquired at $\mathrm{NPR}_{\mathrm{s}}=\mathbf{1 . 0 5}$. 


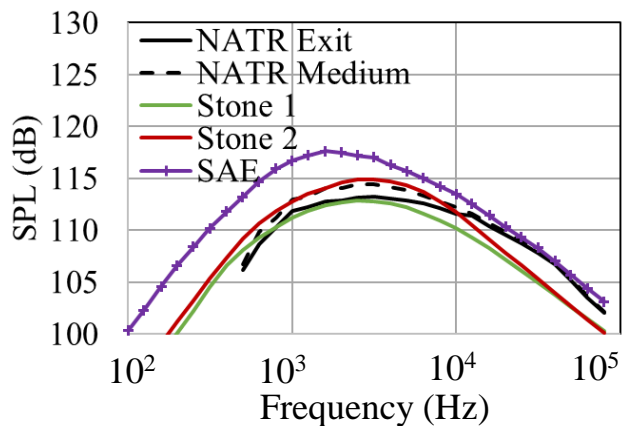

(a)

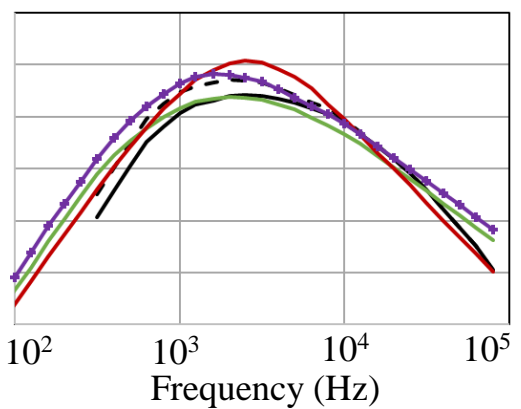

(b)

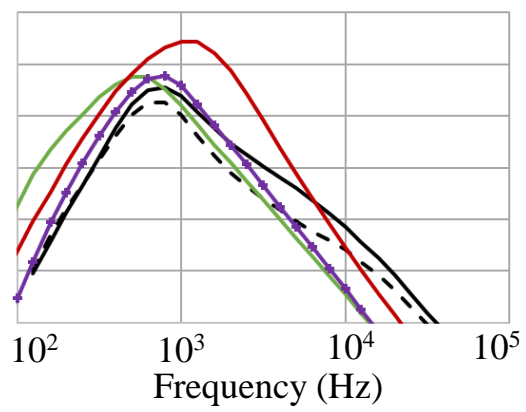

(c)

Figure 13. The one-third octave spectra from ANOPP predictions and measurements at NPR $=1.6$ for emission angles equal to (a) $70^{\circ}$, (b) $110^{\circ}$, and (c) $150^{\circ}$. The NATR spectra are from averaged data and acquired at NPR. $=1.05$.

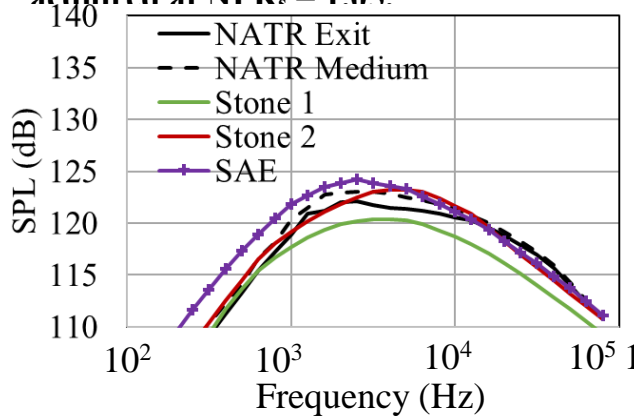

(a)

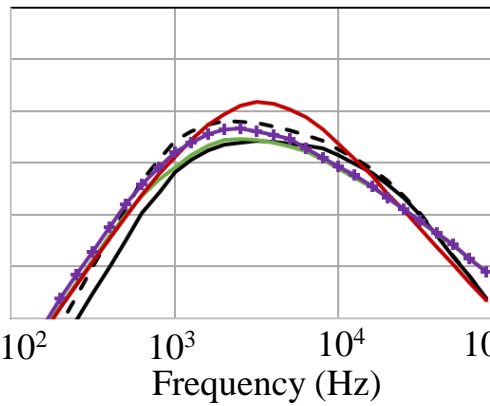

(b)

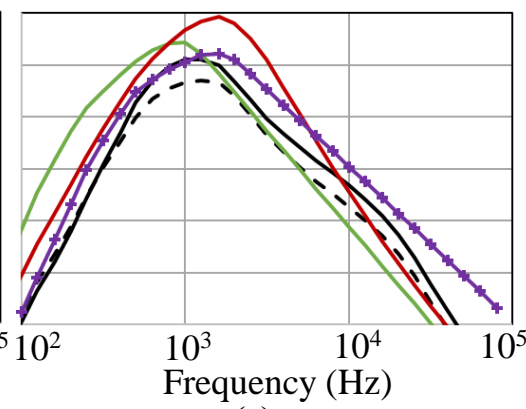

(c)

Figure 14. The one-third octave spectra from ANOPP predictions and measurements at NPR $=2.0$ for emission angles equal to (a) $70^{\circ}$, (b) $110^{\circ}$, and (c) $150^{\circ}$. The NATR spectra are from averaged data and acquired at $\mathrm{NPR}_{\mathrm{s}}=1.05$.

\section{B. Comparisons with Flight Data}

The flight data were compared with predictions using source models within ANOPP and scale-model data. The source levels were propagated using the atmospheric conditions recorded for the flight test. A hard surface was assumed for the ground reflections, which is reasonable for microphones on steel plates surrounded by dirt terrain. The SAE lateral attenuation method was not used for the left and right microphone arrays as a better comparison was achieved between flight data and predictions without attenuation. 


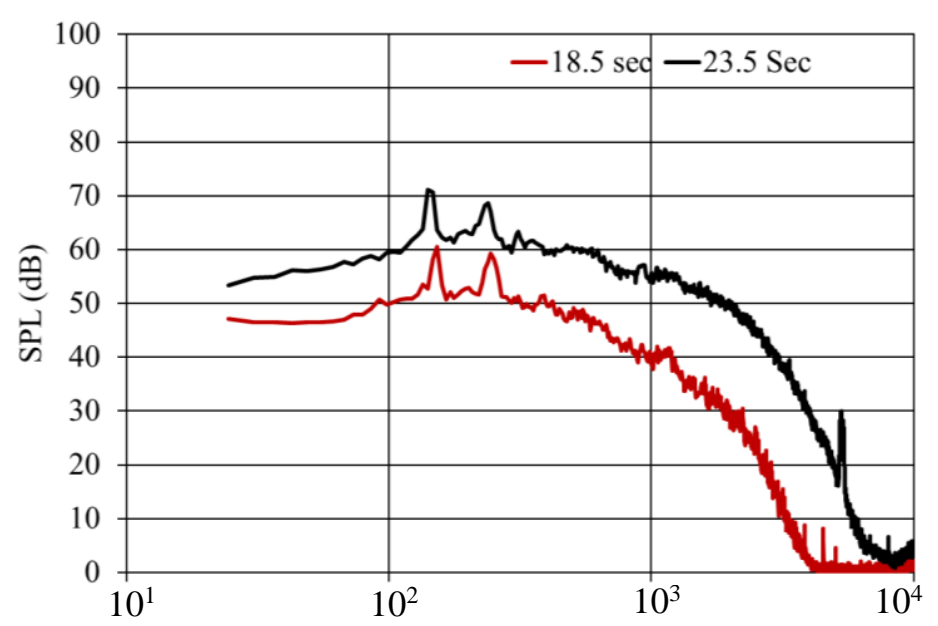

Figure 15. The narrowband flyover spectra acquired at the indicated times for the center microphone and $E P R=1.8$.

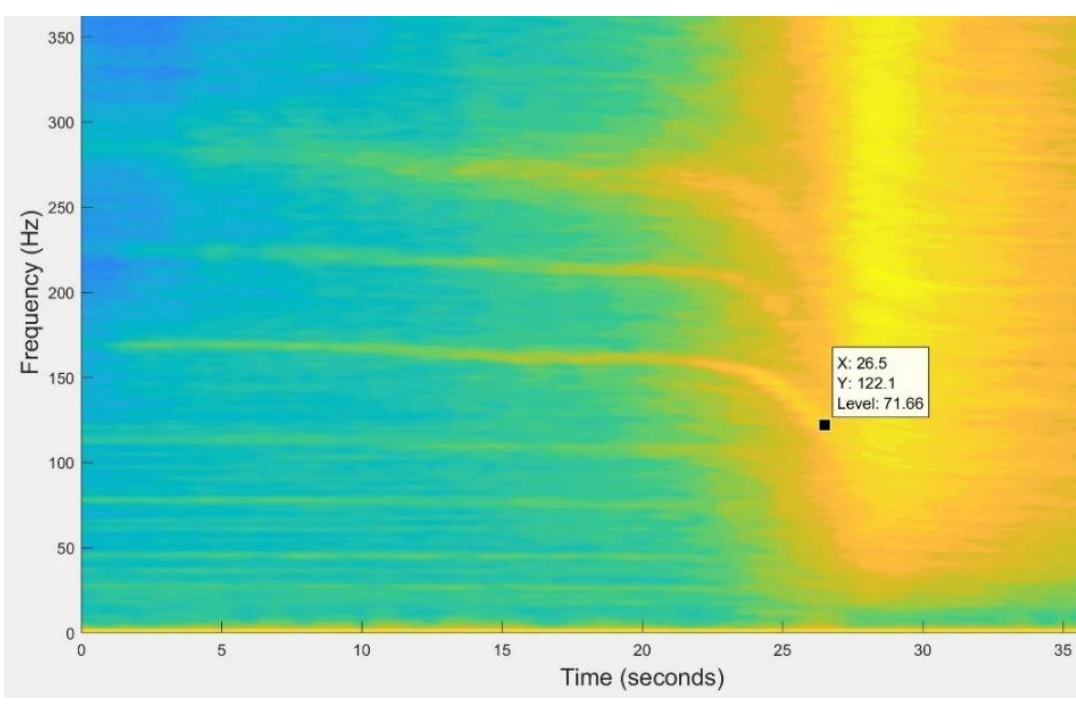

Figure 16. Contours of Learjet flyover SPL as a function of frequency and time for $\mathrm{EPR}=\mathbf{1 . 8}$ for the center microphones.
There were no aircraft position data available from the flight tests, so the alignment between predictions and the flyover data had to be approximated. Since there were compressor tones in the flyover data, it was possible to use the Doppler shift to estimate when the aircraft passed overhead. A sample spectra is shown in Fig. 15. The number of samples for the FFT was increased to 16384 , giving a bandwidth of $1.53 \mathrm{~Hz}$ to make the tones easier to find in the narrowband spectra. Figure 16 shows contours of SPL for frequency versus time for a representative flyover condition. There are several tones between 100 and $300 \mathrm{~Hz}$ where a rapid decrease followed by a leveling out of frequency can be observed between 20 and 30 seconds. A marker in the Figure locates the approximate midpoint of the flyover event. The ANOPP predictions were time-shifted to align with Learjet flyover data using the midpoint flyover event.

The flyover data obtained from the center array for $\mathrm{EPR}=1.8$ are shown in Fig. 17. While the data were acquired at slightly different ambient conditions and slightly different ground speeds (see Table 2), the data repeatability is good for flyover times between 20 and 30 seconds. There is a slight spread in the data for flight times greater than 30 seconds which are within the $10 \mathrm{~dB}$ down point and, therefore, contribute to EPNL calculations. The one-third octave and narrowband data for points 1,2 , and 3 indicated in Fig. 17 are shown in Fig. 18. The tones and broadband

"humps" in the data are not associated with jet noise and, therefore, cannot be predicted by the jet-noise models in ANOPP or by the scale-model data. The greater spread in the PNLs for flight times greater than 30 seconds appears to be associated in part with differences in the broadband hump shown in the narrowband data of Fig. 18 (c). Narrowband and one-third octave comparisons at the peak PNL [Fig. 18 (b)] are quite good between the different runs. For one of the runs (Run 135) the tones and humps indicated in Fig. 18 were removed and the resulting onethird octave spectra on the left side of Fig. 18 were obtained. The tone removal was an effort to determine the impact of the tones and humps on the resulting EPNLs which will be discussed later. The tones were removed by linearly interpolating the acoustic levels between data points on the lower and upper frequency of the tone or broadband hump. For this exercise, the sound pressure levels were replaced with linearly interpolated levels for the same frequency range at all flight times. It is possible that the tone removal procedure did not fully account for the impact of the tones and humps on the resulting spectra and, therefore, the resulting EPNL for the tone removed data can be expected to be somewhat higher than those for the predictions.

The PNLs for the flight data taken with the center array with EPR 1.8 and ANOPP predictions using the SAE, Stone 2, and scale-model data for NPR $=1.8$ are shown in Fig. 19. Tones have not been removed from the flight data (Run 134). The scale-model data are averaged spectra for $\mathrm{NPR}_{\mathrm{s}}=1.05$. The peak PNL level in Fig. 19 for the flight data is close to that of Stone 2 and is higher than those predicted by SAE and the scale-model data. The PNL levels for SAE and the scale model data are similar. 
The spectra corresponding to points 1, 2, and 3 in Fig. 19 are shown in Fig. 20. The tone removed spectra for Run 134 as well as an SAE prediction with a temperature increased by $100^{\circ} \mathrm{F}$ (designated SAE +100 ) have been included in the right column of Fig. 20. The predictions for SAE with the increased temperature have been included to determine the impact of temperature as the temperatures where not recorded during the flyover tests. At 26 seconds [Fig. 20 (a)] SAE, Stone 1, NATR Medium, and Run 134 spectra compare reasonably well except at high frequencies. Peak levels for NATR Exit are slightly lower than those for the flight data and predictions. The peak levels at the peak PNL [Fig. 20 (b)] are similar for Stone 2 and Run 134 while SAE and NATR levels are rough 5 $\mathrm{dB}$ below that for Run 134. The peak level for SAE+100 is slightly less than $2 \mathrm{~dB}$ below that for Run 134 Tone Removed. At 31.5 seconds [Fig. 20 (c)] where the flyover data has a significant hump in the narrowband spectra [see Fig. 18 (c)], the peak levels for Stone 2, SAE, and NATR are well below that for Run 134. The tone removed flyover data has a peak level that is roughly $3 \mathrm{~dB}$ above that for $\mathrm{SAE}+100$. It should also be noted that the 31.5 second flyover time corresponds to a $158^{\circ}$ emission angle, an angle that has a reasonably large shear layer correction for data acquired in a free-jet scale-model facility.

The PNLs for EPR = 1.8 and the left array are shown in Fig. 21. The spread in the data at the peak PNL is greater than that associated with the center microphone array. The increased spread in the data is due to the smaller number of data samples that were available for averaging spectra relative to that for the center array. When a similar number of samples were used for the center array, the spread in peak PNL levels were similar to those in Fig. 21. The impact of the number of samples on the EPNL will be discussed later.

The PNLs for EPR $=1.6$ and the centerline array as well at the ANOPP predictions for NPR $=1.6$ are shown in Fig. 22. Stone 2 slightly underpredicts peak PNL. The peak PNL level for SAE is closer to that for Stone 2 and for $\mathrm{EPR}=1.8$. The NATR data produces the lowest peak PNL levels.

The spectra corresponding to the points 1, 2, and 3 in Fig. 22 are shown in Fig. 23. The one-third octave spectra for the flight data, SAE, Stone 2, and the NATR data are in the right column and the narrowband sound pressure levels for the flight data are in the left column. Comparing the narrowband data of Figs. 23 (a) and 18 (a) shows that the hump in the data at 16.5 seconds for EPR $=1.6$ is not as elevated relative to the broadband levels as that at 26 seconds for EPR $=1.8$ due to increases in the broadband levels with the increased jet velocity. The hump that was present at 31.3 seconds for EPR $=1.8$ [Fig. 18 (c)] is absent from the spectra at 21 seconds for EPR $=1.6$ [Fig. 23 (c)] possibly due to the landing gear being deployed for EPR $=1.8$ and not for $E P R=1.6$. The emission angles were similar for Figs. 18 (c) $\left(158^{\circ}\right)$ and 23 (c) $\left(154^{\circ}\right)$. Stone 2 underpredicts and SAE overpredicts the broadband peak spectral levels at 16.5 seconds. The peak levels for NATR Medium are close to the broadband peak levels. At the peak PNL flyover time, SAE and NATR produce similar peak levels which are well below those of the flight data. Stone 2 produces levels that are close to the flyover peak levels. At 21 seconds, SAE and NATR Exit spectra are similar and have peak levels well below those of the flight data. Tones were not subtracted from the flight data at $\mathrm{EPR}=1.6$ and there is some evidence of a tone at 21 seconds in the flight data.

The PNLs for EPR $=2.0$ and the centerline array along with the ANOPP predictions for NPR $=2.0$ are shown in Fig. 24. The corresponding spectra are shown in Fig. 25. The agreements between the predicted and measured spectra at 22 seconds and at the peak PNL flyover time are similar to those at 26 seconds and at the peak PNL time, respectively, for EPR $=1.8$. The hump in the narrowband data at 28 seconds is similar to that observed in the data at $\mathrm{EPR}=1.8$. Note that the landing gear was deployed for the $\mathrm{EPR}=1.8$ and 2.0 flyovers. Stone 2 produces levels that are closest to the measured data for 28 seconds although the data clearly contains noise components not related to jet noise. 

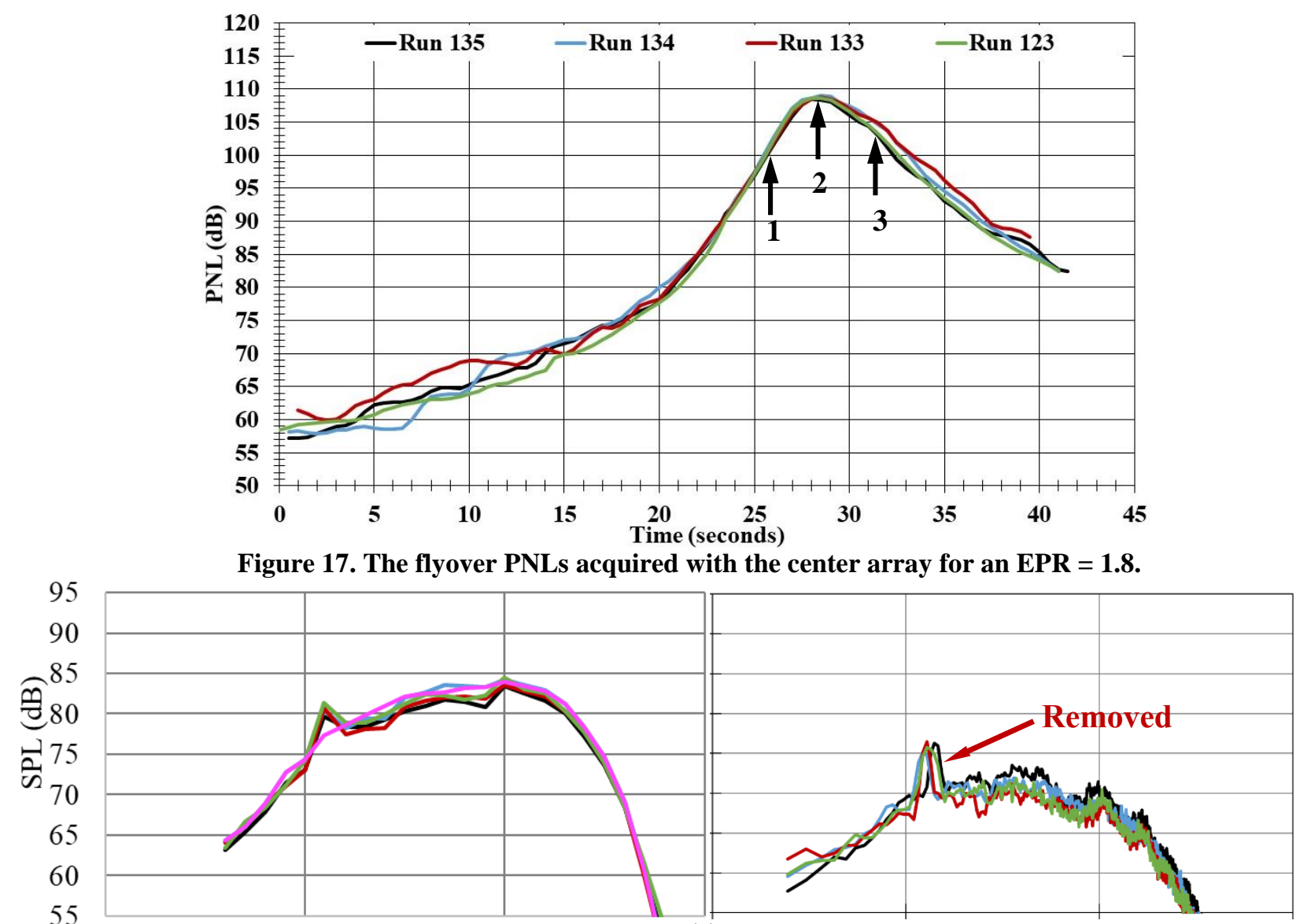

(a)
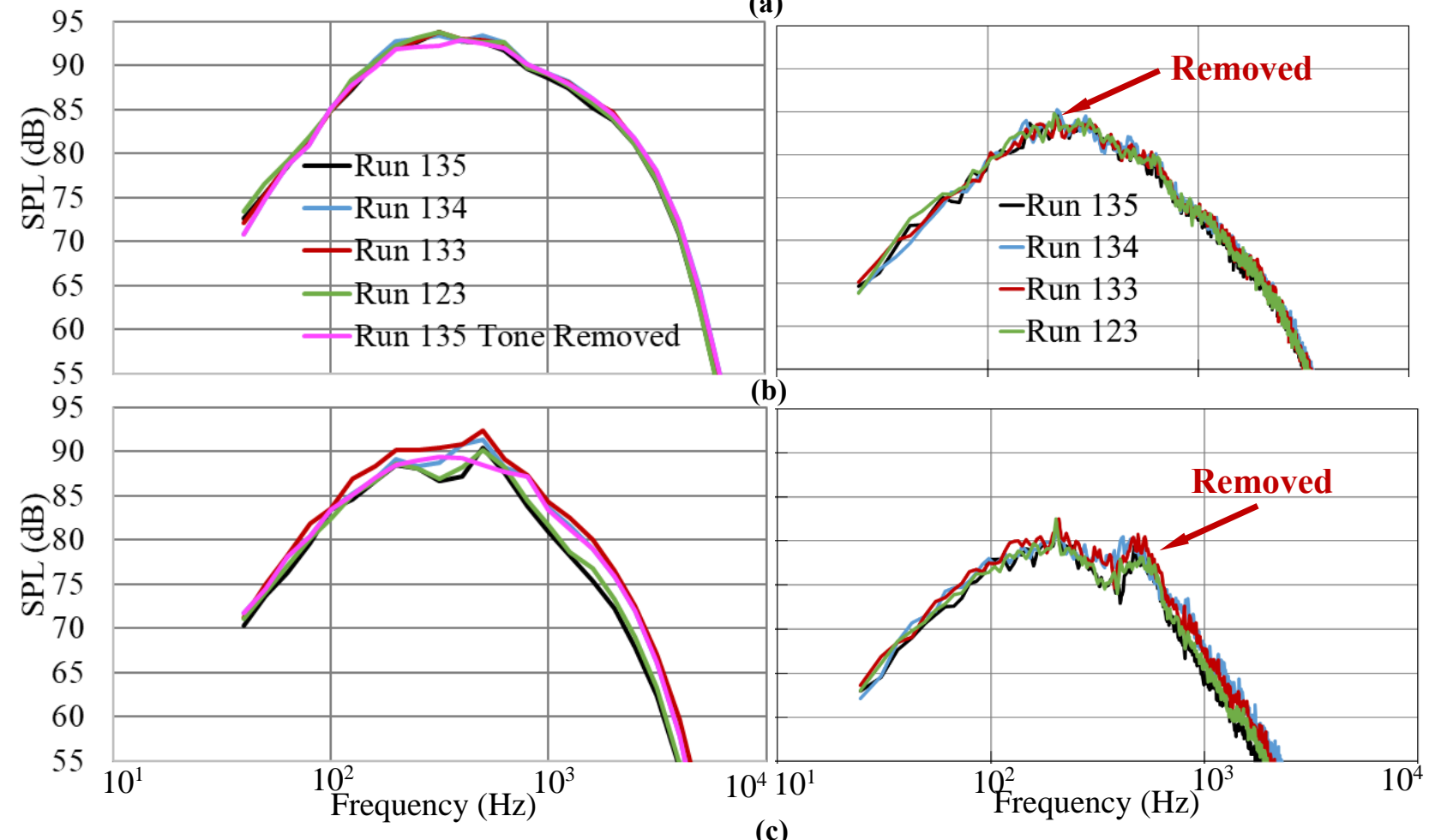

Figure 18. The one-third octave spectra (left) and narrowband spectra (right) for the flyover data (center array) acquired at EPR = 1.8. The data are for (a) $26 \mathrm{sec}$, (b) $28.5 \mathrm{sec}$, and (c) $31.5 \mathrm{sec}$ (see 1, 2, and 3 in Fig. 18). 


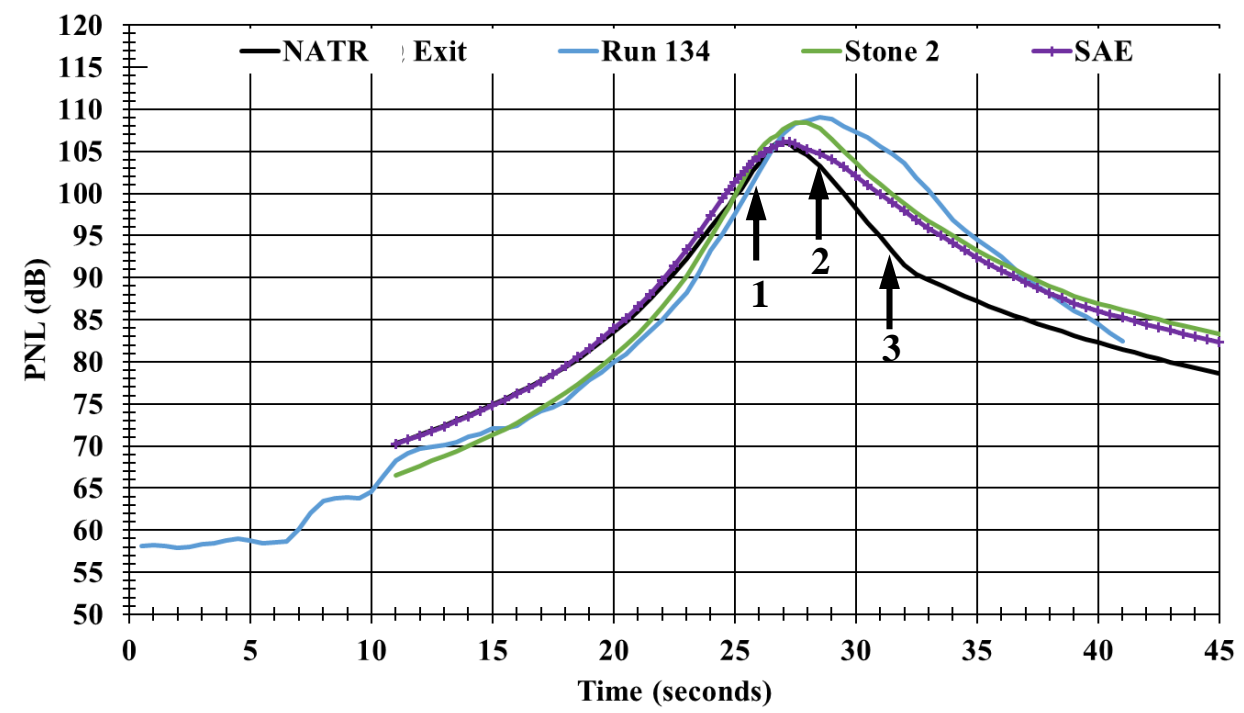

Figure 19. The PNLs at EPR $=1.8$ for the flight data (center array) and NPR $=1.8$ for the ANOPP predictions.
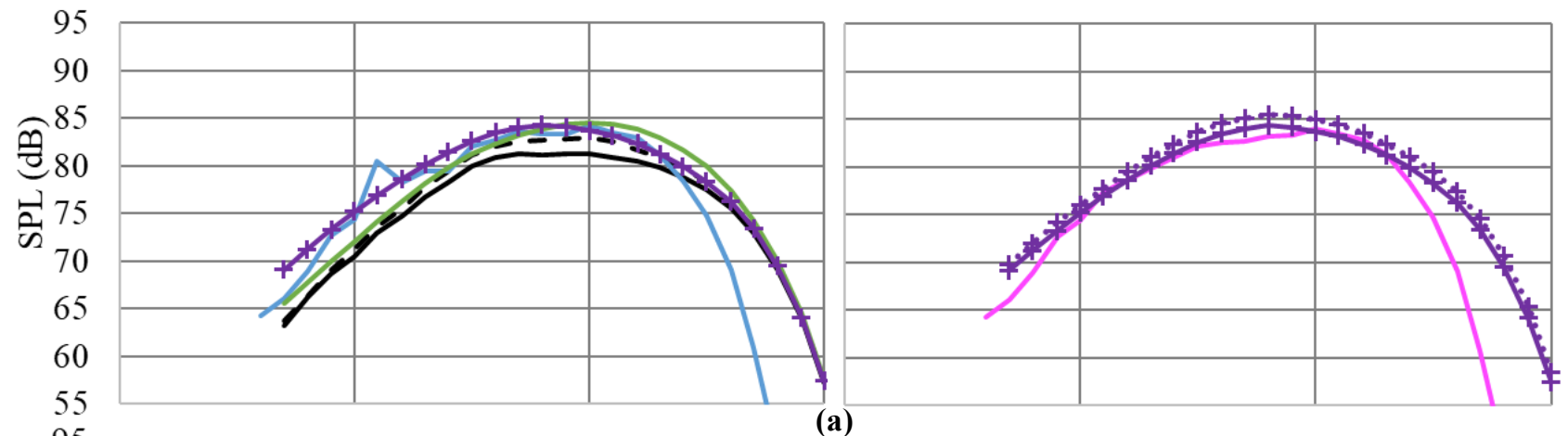

(a)
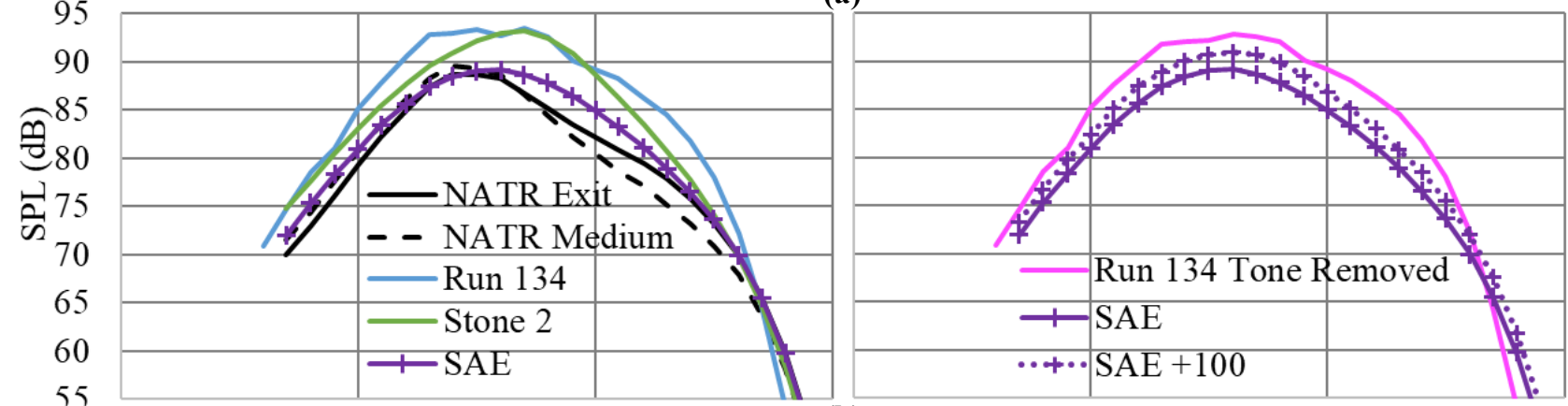

(b)


(c)

Figure 20. The one-third octave spectra for flyover Run 134 (center array) at EPR $=1.8$ and ANOPP predictions at $\mathrm{NPR}=1.8$. The data and predictions are for (a) $26 \mathrm{sec}$, (b) $28.5 \mathrm{sec}$, and (c) $31.5 \mathrm{sec}$ (see 1, 2, and 3 in Fig. 20). 


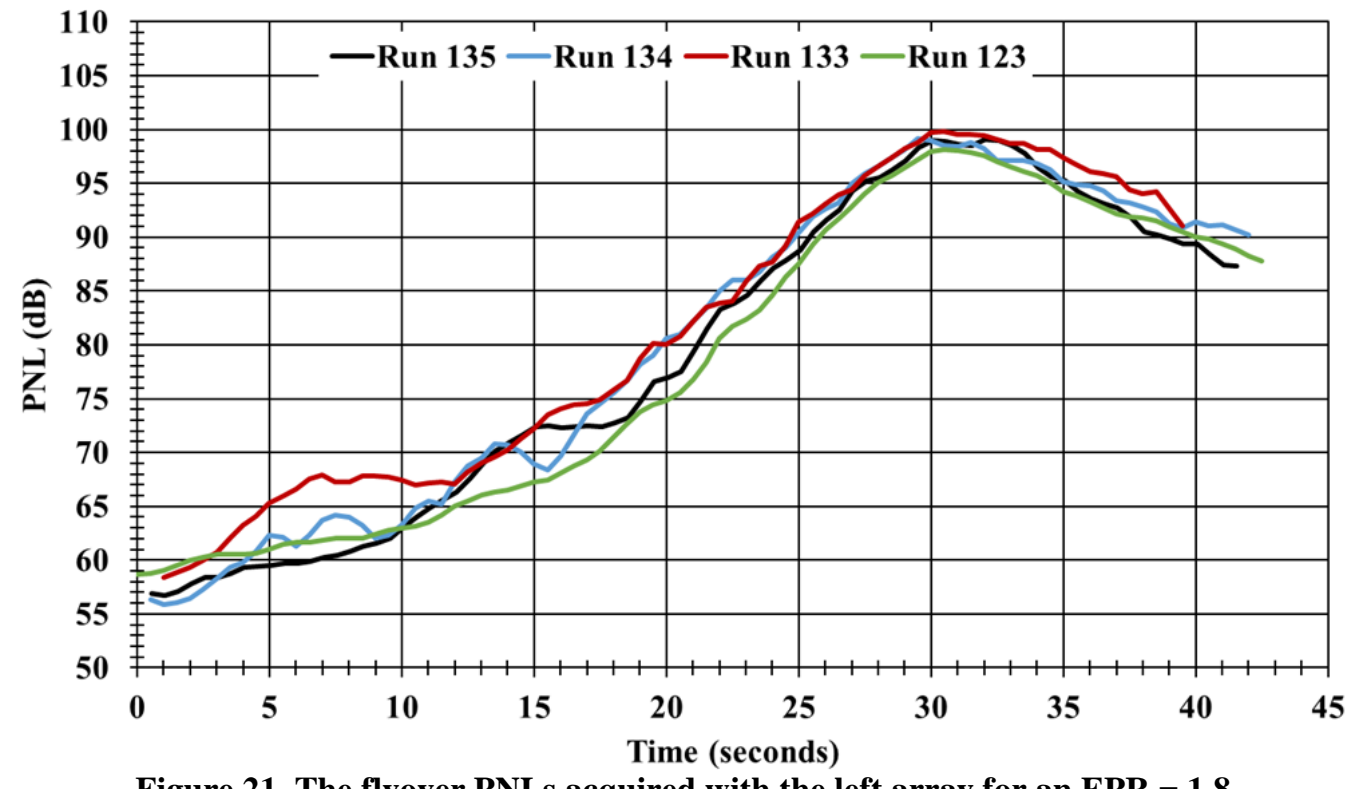

Figure 21. The flyover PNLs acquired with the left array for an $E P R=1.8$.

The effective perceived noise levels (EPNL) were computed for the predictions, scale-model data, and flight data. The values were obtained for a 500' constant altitude flyover over the centerline array at the conditions identified in the flight test matrix (Table 2). The EPNLs were also computed from the data acquired with the left and right microphone arrays. Note that the 500' flyover does not represent a takeoff trajectory and, therefore, the values should not be compared to certification levels.

Table 3. EPNLs for the NATR

\begin{tabular}{|c|c|c|c|}
\hline EPR/NPR & $\begin{array}{c}\text { Flyover } \\
\text { Run }\end{array}$ & $\begin{array}{c}\text { NATR } \\
\text { Exit }\end{array}$ & $\begin{array}{c}\text { NATR } \\
\text { Medium }\end{array}$ \\
\hline \multirow{2}{*}{1.6} & 139 & 92.6 & 92.8 \\
& 140 & 92.7 & 92.8 \\
\hline \multirow{3}{*}{1.8} & 123 & 100.5 & 100.6 \\
& 133 & 100.9 & 100.9 \\
& 134 & 100.9 & 101 \\
& 135 & 100.6 & 100.8 \\
\hline \multirow{2}{*}{2.0} & 121 & 104.5 & 104.6 \\
& 130 & 105.0 & 105.1 \\
\hline
\end{tabular}

The impact of the shear layer correction assumptions on EPNL is summarized in Table 3 . The results show that the source distribution assumptions within the shear layer correction impact the EPNLs by no more than 0.2 EPNdB for all of the runs in Table 3.

Figure 26 (a) shows the EPNL noise levels for the center microphone flyover. The difference in EPNL of the predictions and the NATR data relative to the flight data are shown in Figure 26 (b). Positive values mean the EPNL is lower than the flight data. For all runs and EPRs, the flight EPNLs are higher than the levels for SAE, Stone 2, and NATR Exit by 1 to $5 \mathrm{EPNdB}$. The Stone Jet 2 prediction are the closest to the flight data and are within 1 to 2 EPNdB of the flight data. The NATR data is furthest from the flight data, with differences ranging from approximately 3 to $5 \mathrm{EPNdB}$. The average of the difference in the flight

EPNL and NATR EPNL at each EPR decreases with increasing EPR.

Figure 27 and Figure 28 show the results from the left and right microphone measurements, respectively. The predictions and NATR data are identical from left to right due to assumed symmetry, where the flight data varies as measured. Since the left engine on the Learjet was used for the tests and the right engine was at idle, there could be differences from installation effects. However the results do not show a preferred direction for propagation due to installation effects. Furthermore, the differences in the flight EPNL are as high as 2 EPNdB between the left and right measurements for the same flyover.

Several studies were done to understand the impact of the tones in the flyover data on the resulting EPNL. The studies focused on the EPR 1.8 data. First, the flight data contains tones that are probably from the compressor of the turbojet engines. They were observed as the Learjet approached the microphones and were manually subtracted from the acoustic spectra at all flyover times. For Run 134, the EPNL reduced by 0.2 EPNdB. Secondly, a "hump" (see Fig. 18) was removed and further reduced the EPNL by an additional 0.3 EPNdB. So obvious non-jet portions of the spectra reduced the EPNL by $0.5 \mathrm{EPNdB}$. It should be noted that the tone subtraction did not entirely remove the impact of non-jet-noise sources on the modified spectra shown by the pink curves in Fig. 18.

The EPNL was also found to be impacted by the number of microphones included in the flyover averaging. There were 8 microphones in the center array and only 6 in the left and right arrays. In several cases, one microphone for the left and right array was not functioning and removed from the averaging. Runs where more than one microphone was not functioning in the array were not included. To investigate the impact of fewer microphones 
in the array, a study was done with the center microphones where the number was reduced from 8 to 5 . Then the EPNL was computed and found to change by 1 EPNdB. So the number of microphones included in the flyover arrays is an important factor for data quality and reducing the uncertainty.

Another unknown from the Learjet data are the actual test conditions for the engine from each run. The engine temperatures and EPRs were recorded from the cockpit gauges during a pre-flight test in Ohio. The EPR of the left engine and the desired flight speed was matched as close as possible during the flyover tests in Arizona, but engine temperatures were not recorded. The SAE prediction model was used to investigate the impact of increasing the engine temperature since the SAE predictions were predominantly lower than the flight data and a higher temperature will increase the jet noise due to increased jet velocity. It was determined that the EPNL for the center microphones can increase by $1.5 \mathrm{EPNdB}$ for each $100^{\circ} \mathrm{F}$ increase in jet exit temperature for an NPR $=1.8$. The preliminary runs conducted in Ohio indicated $54^{\circ} \mathrm{F}$ to $72^{\circ} \mathrm{F}$ differences between the left and right engines running at matched EPR. Therefore there is expected uncertainty associated with determining the actual engine temperatures during the flight test. This is likely one contributing factor for the flight data EPNL being consistently higher than the NATR data and prediction methods.

\section{Conclusions}

System level noise assessments have been made comparing empirical prediction methods, model scale test data and flight data using a Lear 25 aircraft. Jet noise was the primary emphasis of the work with a goal of applying it to future commercial supersonic transport aircraft. A primary objective of the work was to determine the uncertainty associated with noise metrics such as effective perceived noise level. The results show that the flight data is consistently higher in noise levels compared to both predictions and the scaled model test data. This is consistent with findings during the 1970s when predictions models such as the SAE method were developed. The noise levels from the predictions and scale-model data were below those for the flight data by $2.5-3.5,1-2$, and $3-5 \mathrm{EPNdB}$ for the SAE model, the Stone Jet model, and the scale-model data, respectively.

The differences between the flight data EPNLs and those from the predictions and scale model data were investigated. A concern with the flight data was the lack of information about the engine exhaust temperatures and the aircraft position. A $100^{\circ}$ to $200^{\circ} \mathrm{F}$ difference in exhaust gas temperature would be enough to explain the underprediction of the model data and empirical prediction methods. The aircraft position uncertainty contributes to difficulties comparing flyover spectra but not the EPNL assessments. Even though the turbojet powering the Lear 25 is jet noise dominant, there is evidence of compressor tones from the inlet and possibly airframe noise sources in the aft direction that contribute to EPNL calculations and make comparisons challenging.

Analysis of the flyover data showed the importance of using a line of microphones and averaging the acoustic pressures using a time shift. This was shown to impact the EPNL by as much as 1 EPNdB. The averaging process provided sufficient resolution to compute narrowband spectra, which was used to estimate the aircraft position from the Doppler shift of the tones.

The scale-model experiments used a two-stream nozzle replicating the features of the Lear 25 nozzle system. The flow rates for the secondary stream were assumed to be low and the NPR was set equal to roughly that of the flight stream. Increasing the NPR of the secondary stream slightly (from 1.05 to 1.10) had little impact on the acoustic spectra. The source distribution assumptions in the shear layer correction slightly impacted spectral shape and peak radiation angle but did not have a significant impact on EPNLs. Levels in the peak jet-noise direction compared reasonably well with the SAE model and were consistently below those for Stone 2.

There are significant differences between the various empirical jet-noise prediction methods included in ANOPP. The Stone 2 predictions came the closest to agreeing with flight data. The SAE method provided reasonable agreement with the NATR data. Due to the uncertainty in engine conditions during the flight test and the tones present in the spectra, it is difficult to assess which method is really better for this application. Additional work is required to identify the additional noise sources and estimate their contribution to the EPNL as well as quantify the differences in measured levels from the dual-stream nozzle configuration used here and those from a single stream nozzle. 


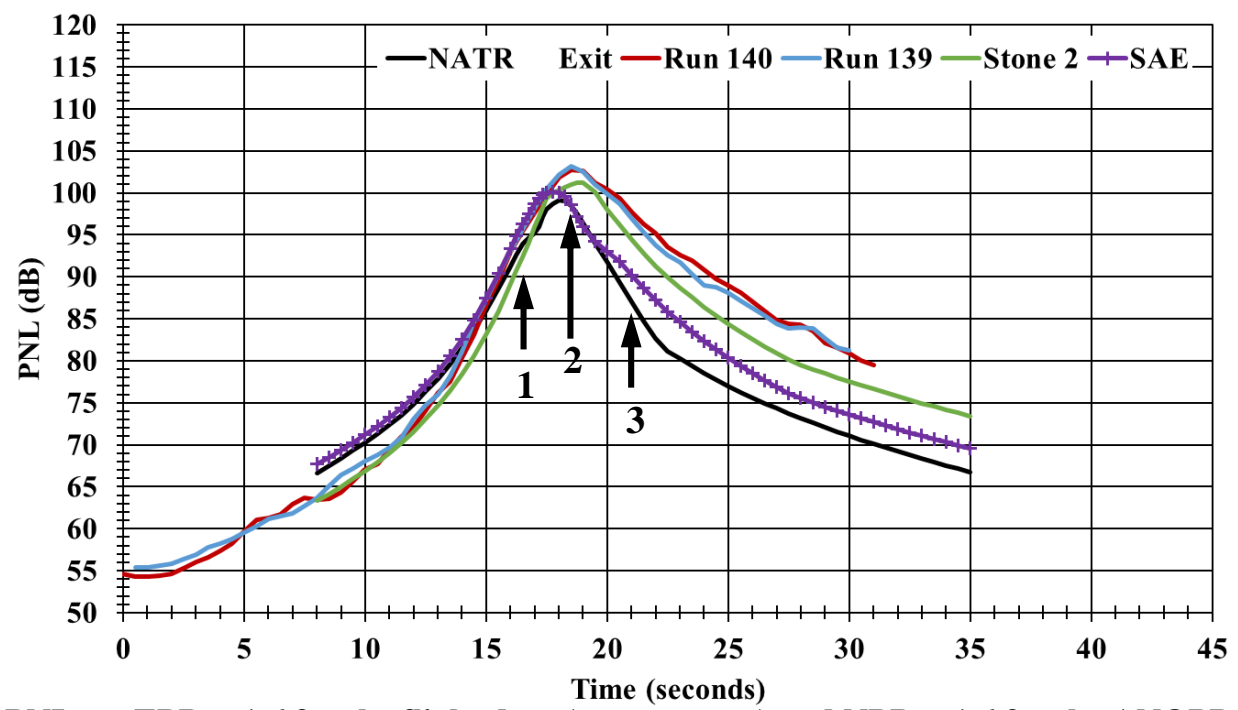

Figure 22. The PNLs at EPR = 1.6 for the flight data (center array) and NPR = 1.6 for the ANOPP predictions.
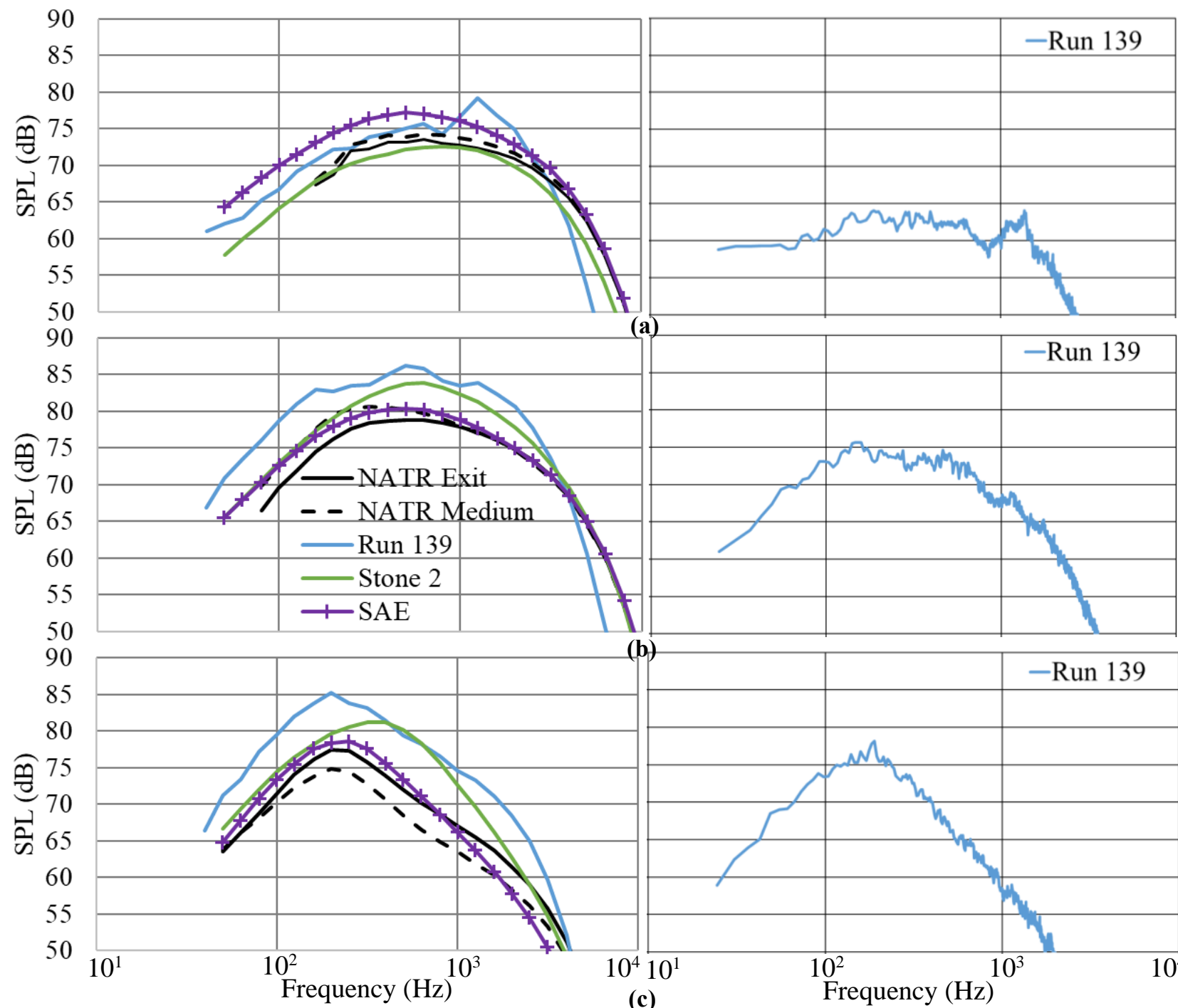

(a)

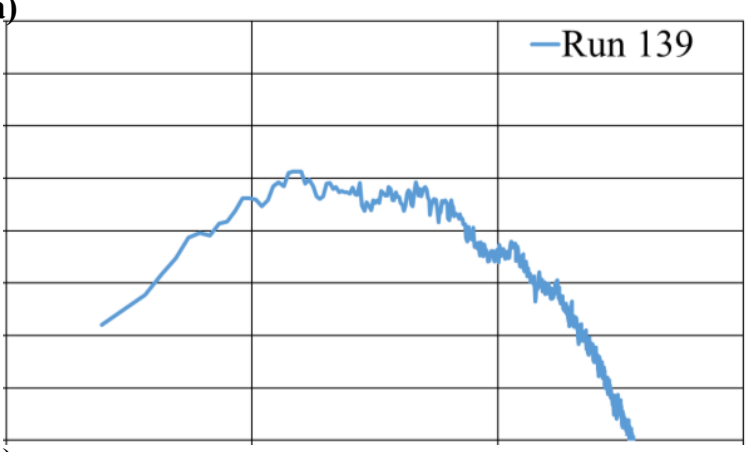

(b)

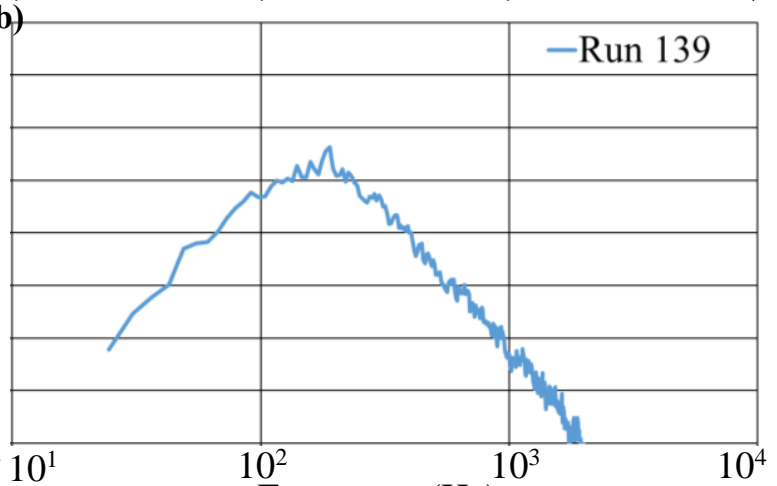

(c)

Frequency $(\mathrm{Hz})$

Figure 23. The one-third octave (left) and narrowband (right) spectra for flyover Run 139 (center array) at EPR = 1.6. The ANOPP predictions at NPR = 1.6 are shown in the left column. The data and predictions are for (a) 16.5 sec, (b) $18.5 \mathrm{sec}$, and (c) $21 \mathrm{sec}$ (see 1, 2, and 3 in Fig 23). $^{23}$. 


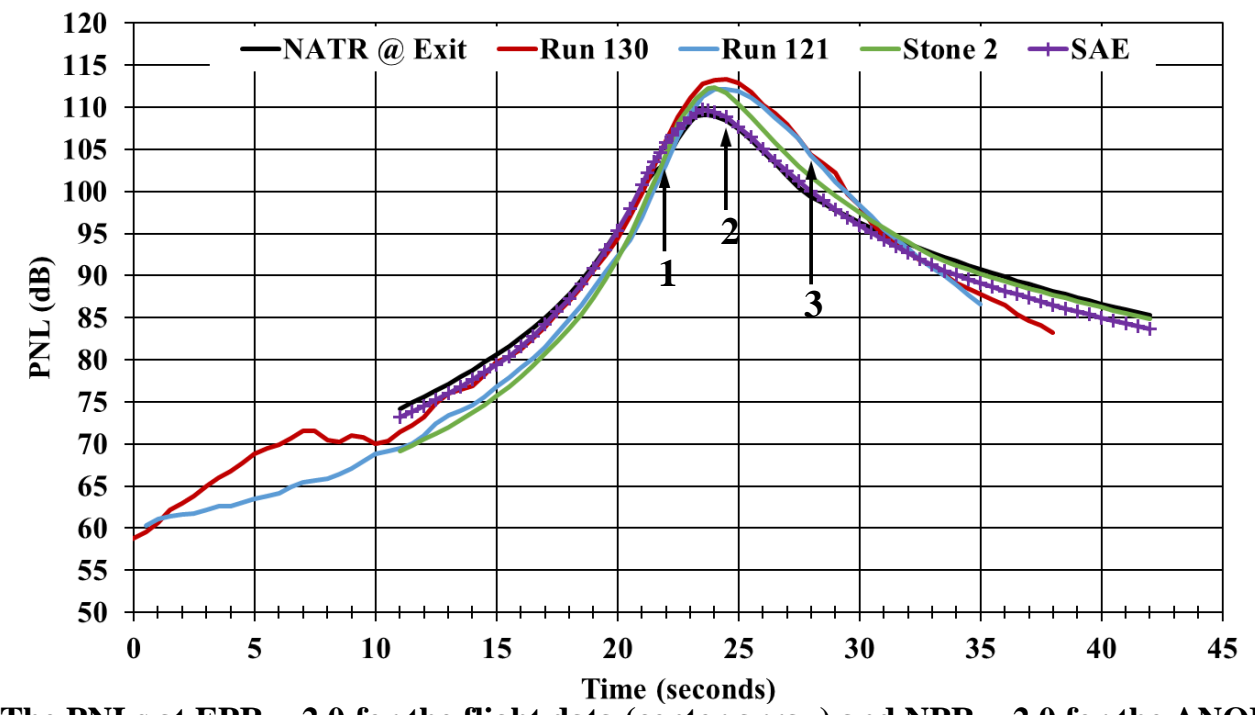

Figure 24. The PNLs at EPR = 2.0 for the flight data (center array) and NPR = 2.0 for the ANOPP predictions.

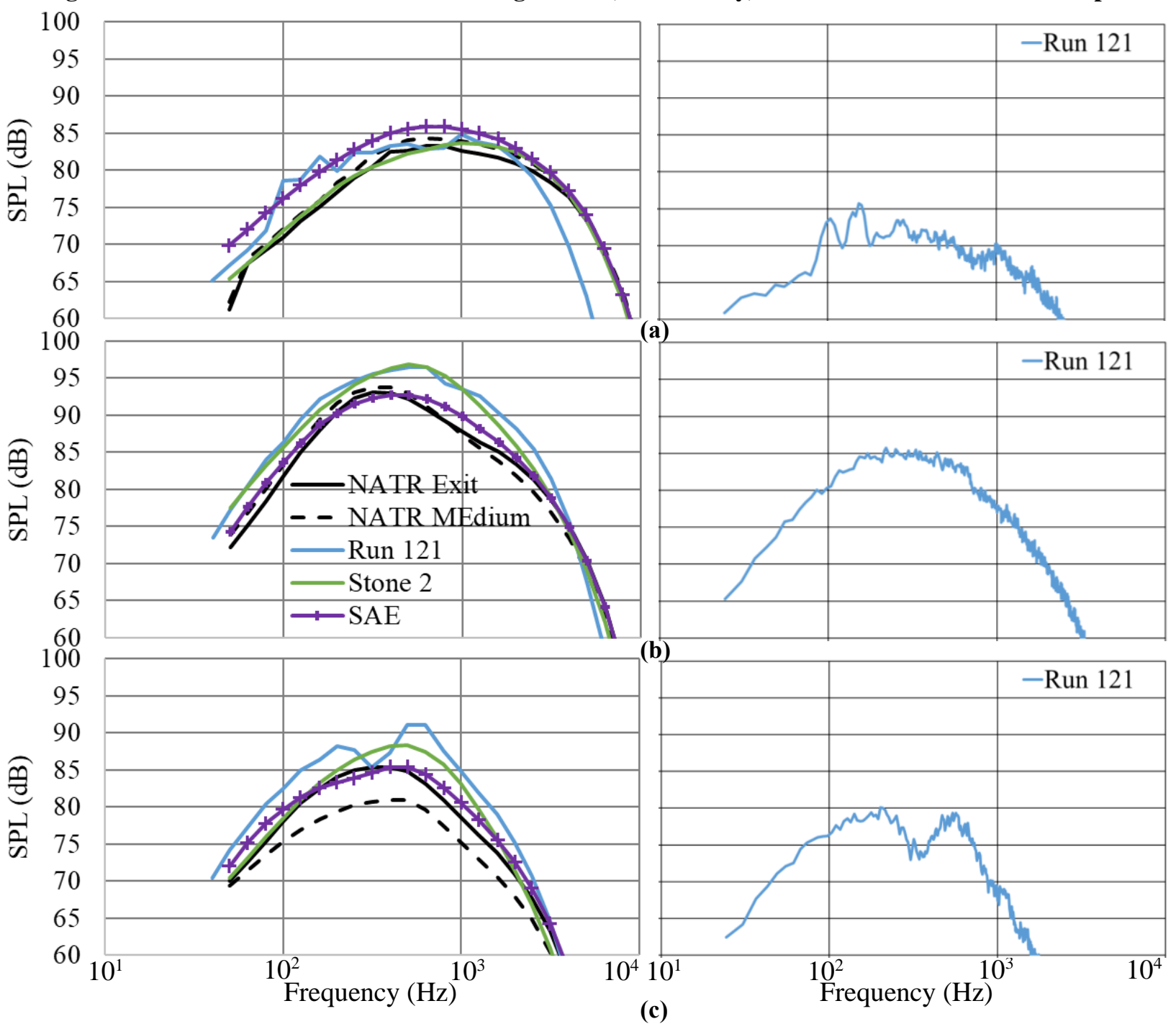

Figure 25. The one-third octave (left) and narrowband (7ight) spectra for flyover Run 139 (center array) at $E P R=2.0$. The ANOPP predictions at NPR =1.6 are shown in the left column. The data and predictions are for (a) $22 \mathrm{sec}$, (b) $24.5 \mathrm{sec}$, and (c) $28 \mathrm{sec}$ (see 1, 2, and 3 in Fig. 25). 


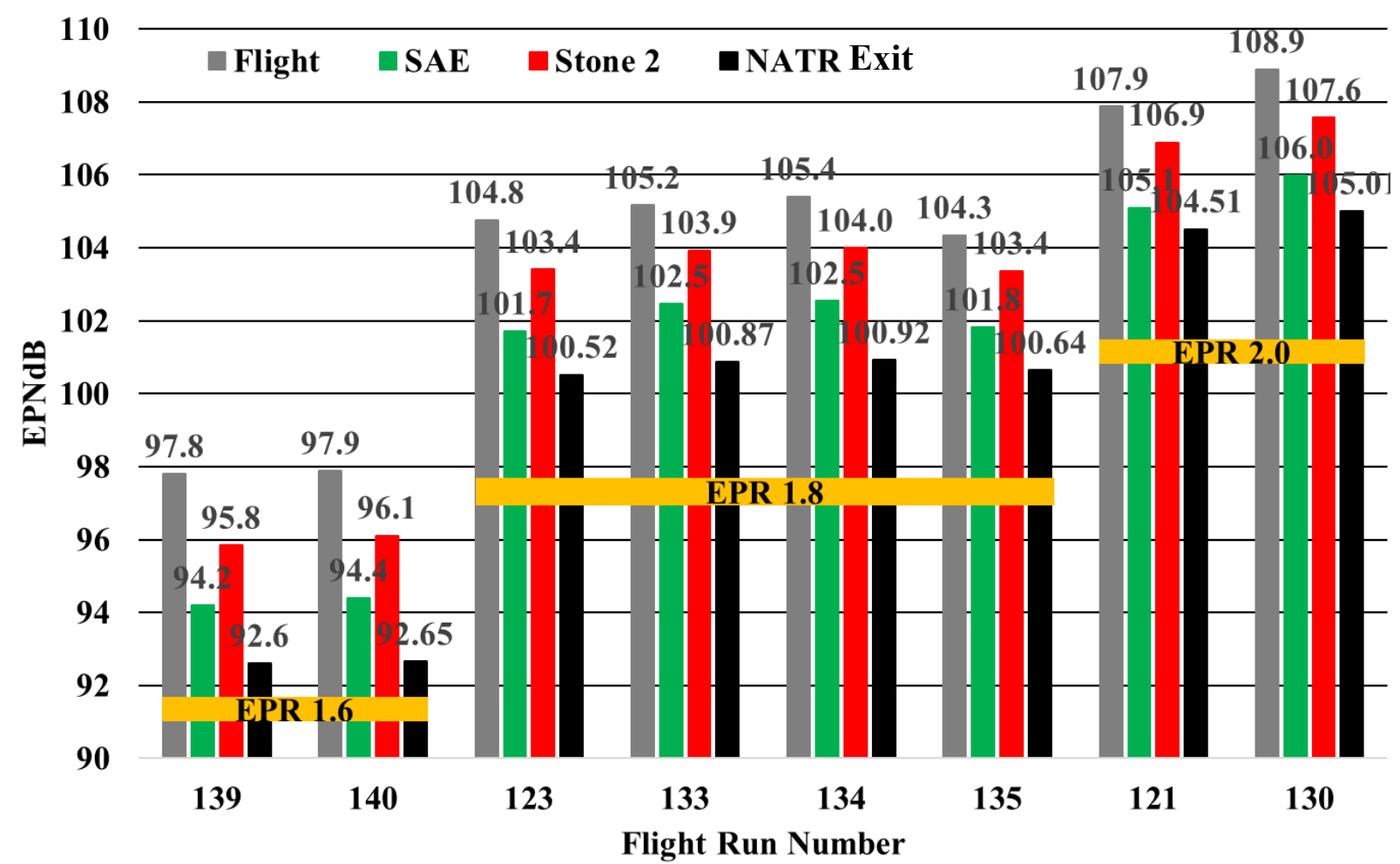

(a)
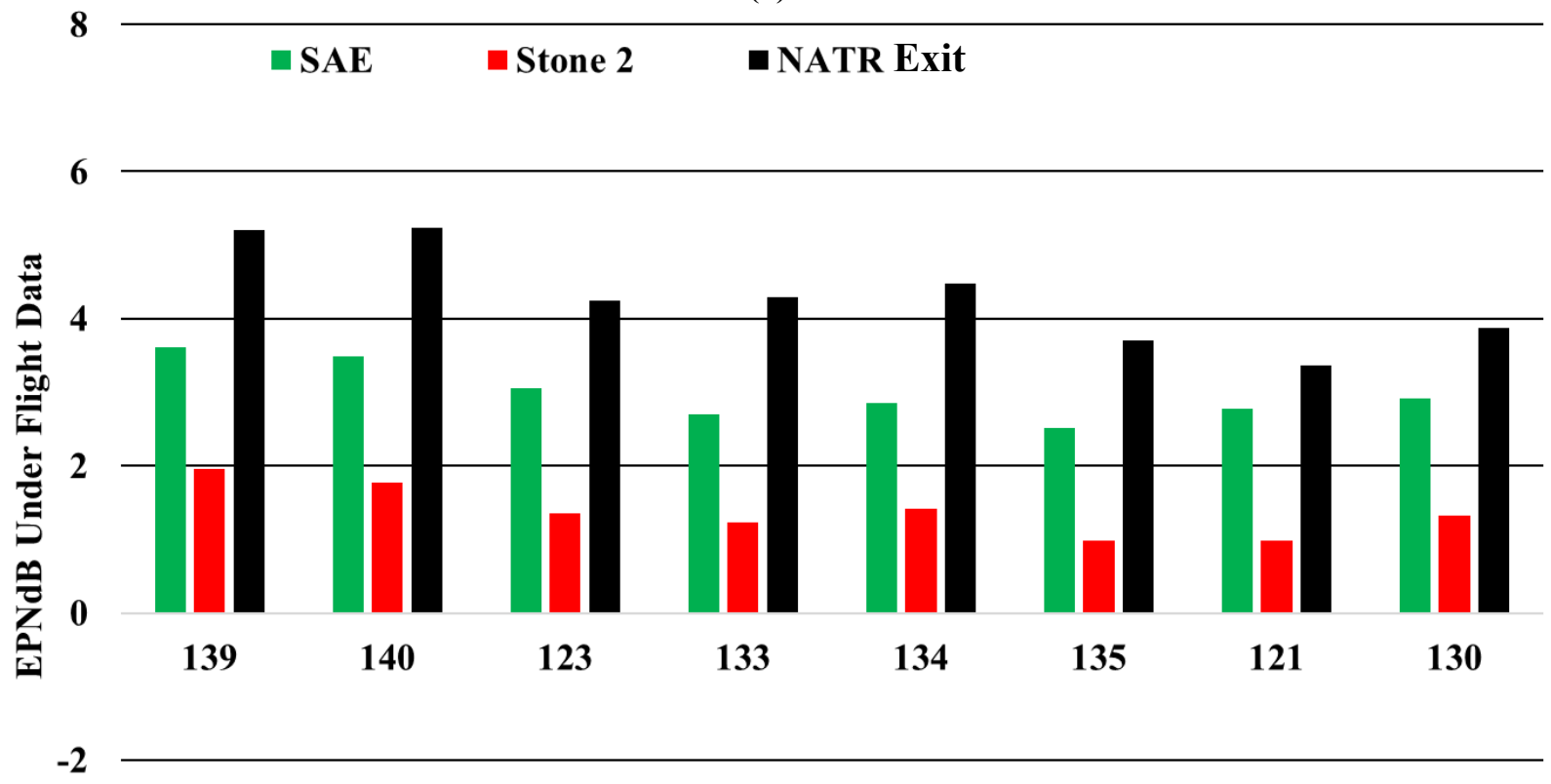

$-4$

\section{Flight Run Number}

(b)

Figure 26. The EPNLs computed for the flyover data measured by the center microphone array, NATR Exit data, SAE predictions, and Stone 2 predictions in (a) and the difference in the predicted and flyover EPNLs in (b). 


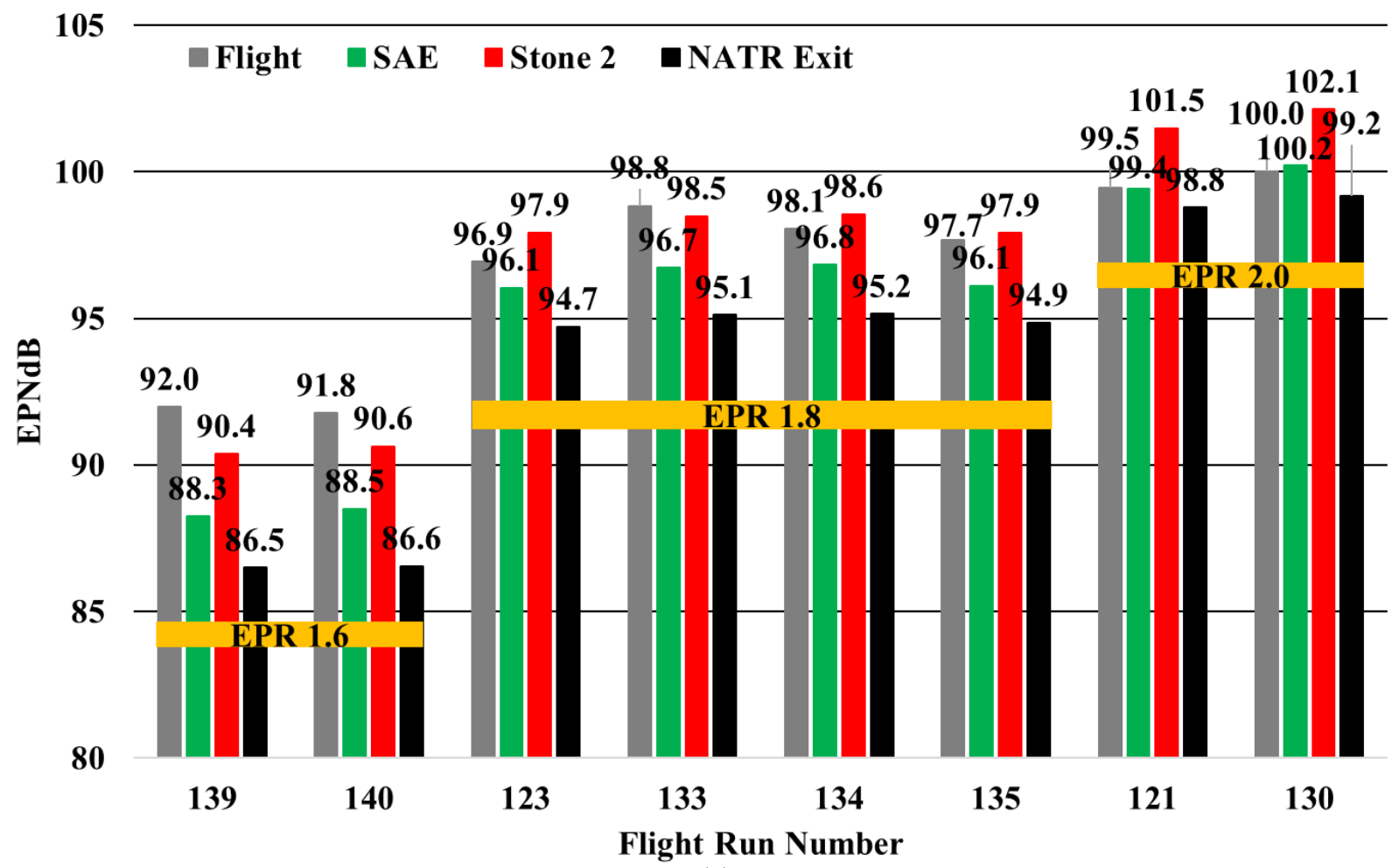

(a)

$$
8
$$

-SAE $\quad$ Stone $2 \quad$ NATR Exit

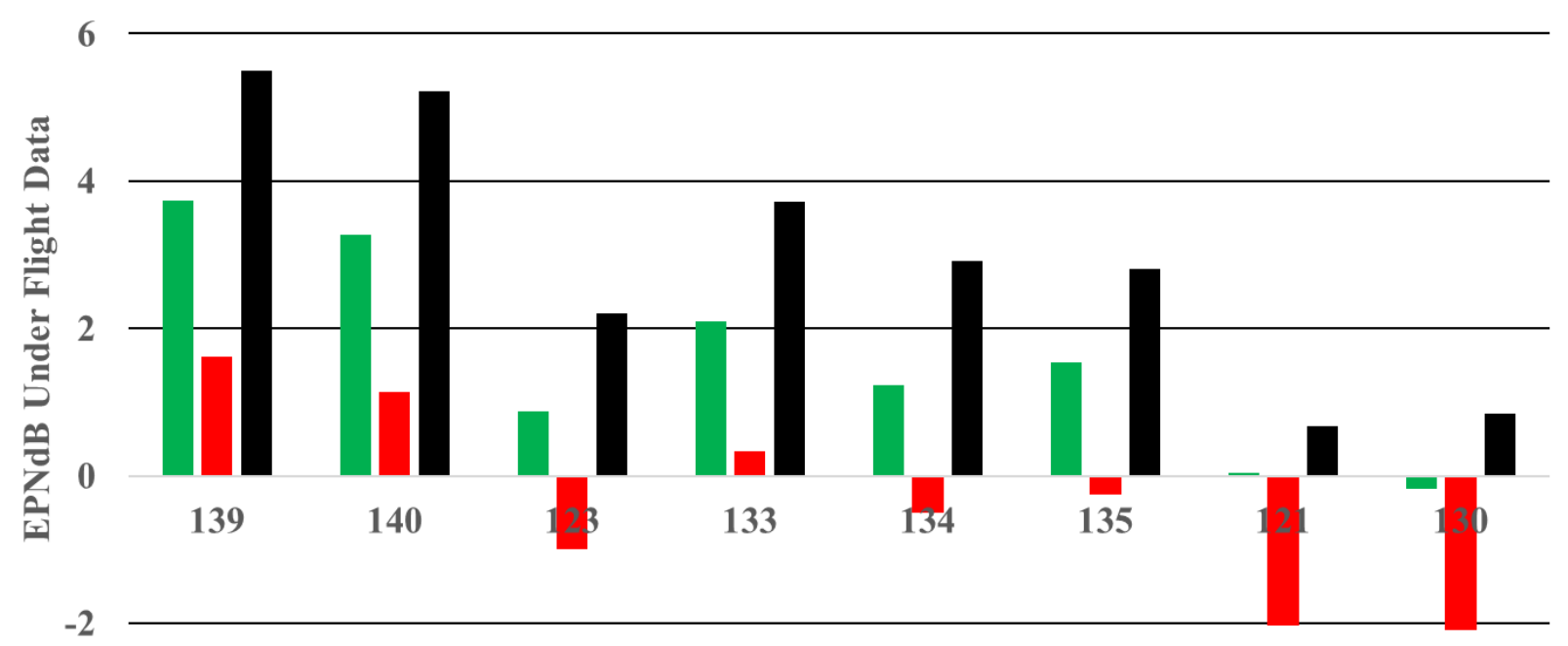

Flight Run Number

(b)

Figure 27. The EPNLs computed for the flyover data measured by the left microphone array, NATR Exit data, SAE predictions, and Stone 2 predictions in (a) and the difference in the predicted and flyover EPNLs in (b). 


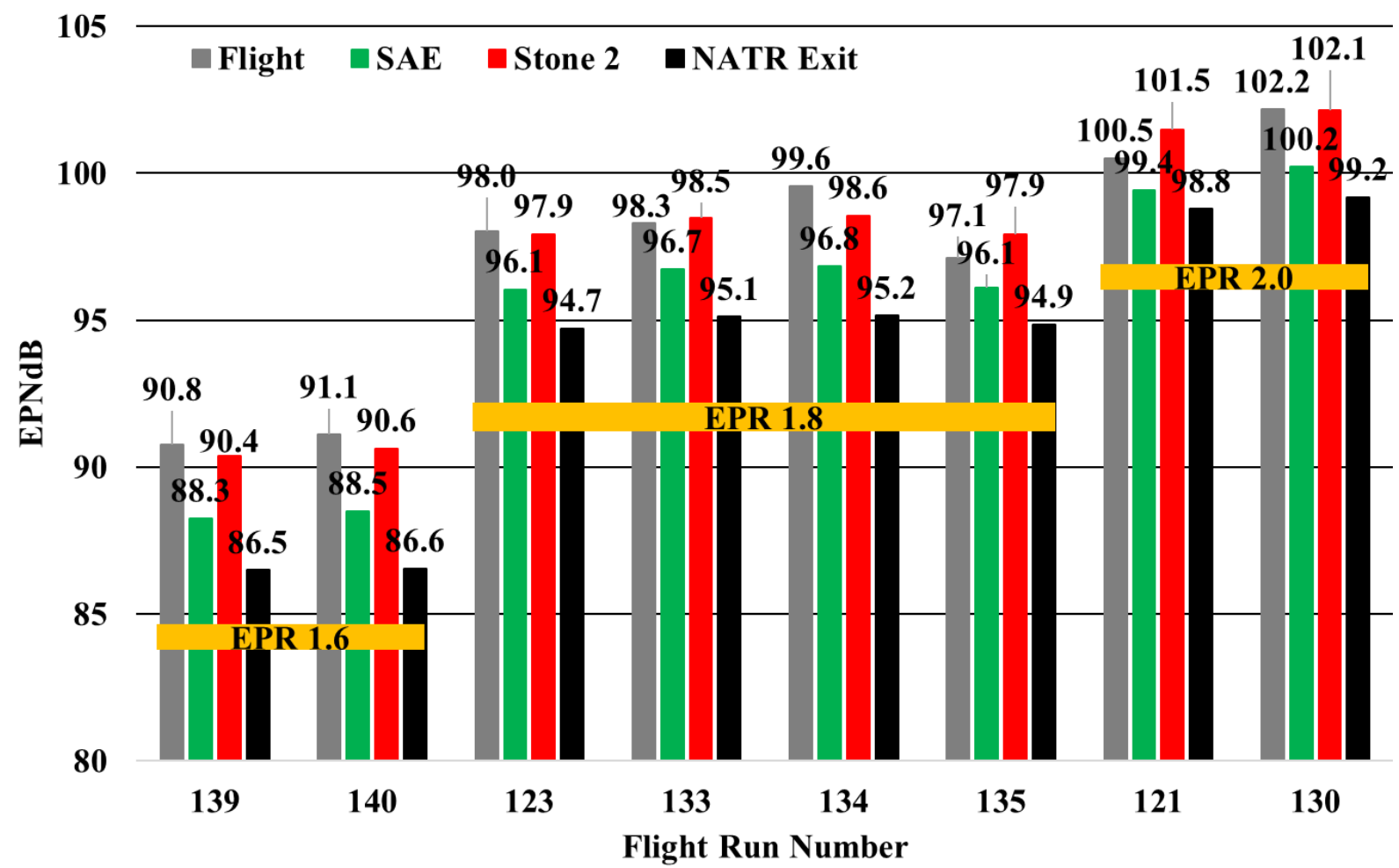

(a)

8
口 SAE
- Stone 2
- NATR Exit

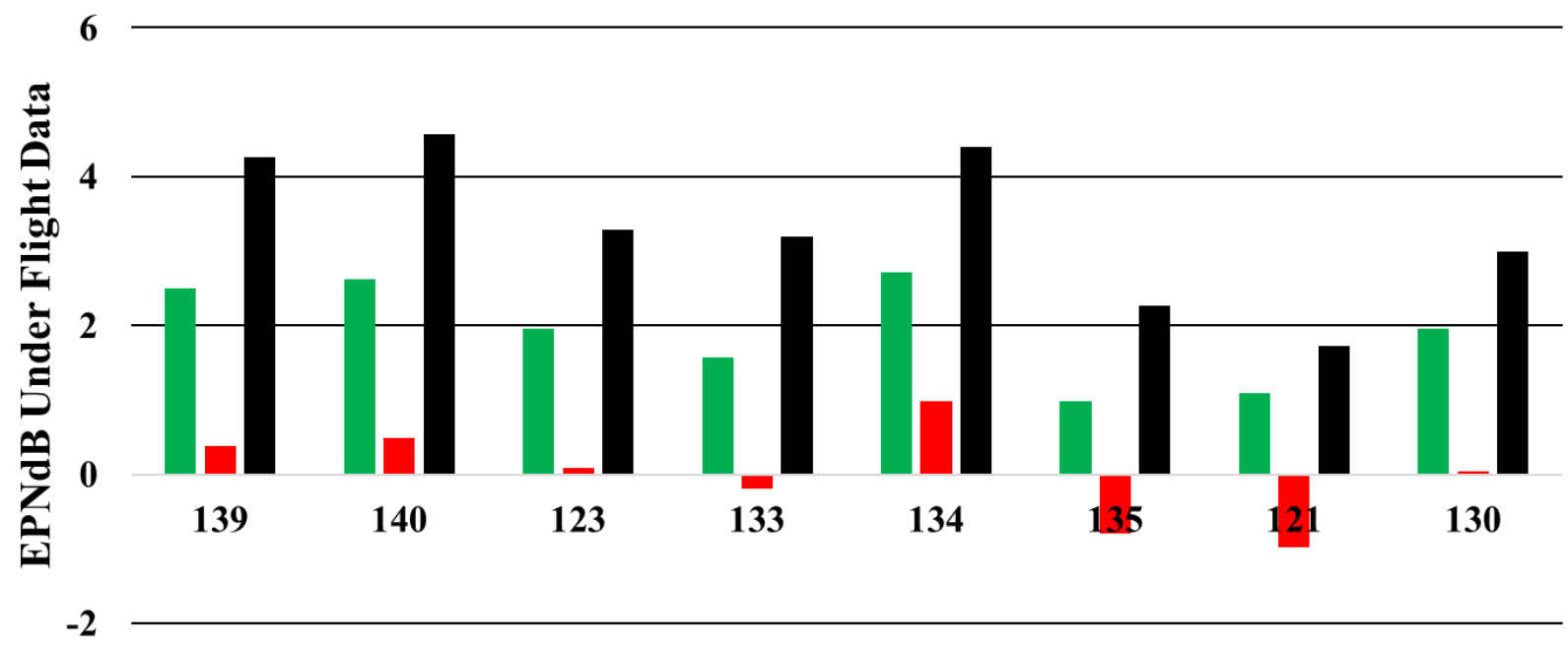

$-4$

\section{Flight Run Number}

(b)

Figure 28. The EPNLs computed for the flyover data measured by the right microphone array, NATR Exit data, SAE predictions, and Stone 2 predictions in (a) and the difference in the predicted and flyover EPNLs in (b). 


\section{Acknowledgements}

The authors thank Charlie Smith of the NASA Langley Research Center for providing the microphone flyover

data from the Learjet flight test. Thanks also goes to Bill Shuster of Honeywell Aerospace for providing the weather balloon atmospheric data from the flight tests, and Cliff Brown of NASA Glenn for helping with the data processing. This work was sponsored by the NASA Commercial Supersonic Technology (CST) project.

\section{References}

${ }^{1}$ Zorumski, W. E., “Aircraft Noise Prediction Program Theoretical Manual, Parts 1 and 2,” NASA TM-83199, 1982; Latest revision: Level 31.

${ }^{2}$ Brown, C. and Bridges, J., "An Analysis of Model Scale Data Transformed to Full Sale Flight Using Chevron Nozzles," NASA TM-2003-212732, 2003.

${ }^{3}$ https://www.aerionsupersonic.com/

${ }^{4}$ https://boomsupersonic.com/

${ }^{5}$ Gas Turbine Jet Exhaust Noise Prediction, SAE Aerospace Recommended Practice ARP 876, Society of Automotive Engineers, March 1978.

${ }^{6}$ Hough, J. and Weir, D., "Small Engine Technology (SET) - Task 13 ANOPP Noise Prediction for Small Engines: Jet, Core, and Turbine Module Revisions,” Allied Signal Engines Report No. 21- 9655, 1997.

${ }^{7}$ Stone, J. R., and Montegani, F. J., "An improved Prediction Method for the Noise Generated in Flight by Circular Jets," NASA TM 81470, 1980.

${ }^{8}$ Stone, J.R., Krejsa, E.A., Clark, B.K. and Berton, J.J., Jet Noise Modeling for Suppressed and Unsuppressed Aircraft in Simulated Flight," NASA TM-2009-215524, March 2009.

${ }^{10}$ Bushell, K. W., "Measurement and Prediction of Jet Noise in Flight," AIAA-75-461, AIAA 2 ${ }^{\text {nd }}$ Aero-Acoustics Conference, Hampton, VA, March 24 - 26, 1975.

${ }^{11}$ Brooks, J. R., "Flight Noise Studies on Turbojet Using Microphones Mounted on A $450 \mathrm{ft}$ Tower," AIAA-77-1325, AIAA $4^{\text {th }}$ Aeroacoustics Conference, Atlanta, GA, October 3 - 5, 1977.

${ }^{12}$ Viswanathan, K., "Improved Method for Prediction of Noise from Single Jets," AIAA Journal, Vol. 45, No. 1, January 2007, pp. 151-161.

${ }^{13}$ Viswanathan, K., and Czech, M. J., "Role of Jet Temperature in Correlating Jet Noise," AIAA Journal, Vol. 47, No. 5, May 2009, pp. $1090-1106$.

${ }^{14}$ Viswanathan, K., and Czech, M. J., "Measurement and Modeling of Effect of Forward Flight on Jet Noise," AIAA Journal, Vol. 49, No. 1, January 2011, pp. 216 - 234.

${ }^{15}$ Preisser, J.S. and Marcolini, M.A., "Flight Acoustic Measurement Techniques and Applications", NASA Langley Research Center internal report.

${ }^{16}$ Soeder, R. H., Wnuk, S. P., and Loew, R. "Aero-Acoustic Propulsion Laboratory Nozzle Acoustic Test Rig User Manual," NASA/TM - 2006-212939, 2006.

${ }^{17}$ ANSI S1.26-1995 (R2004), "Method for the Calculation of the Absorption of Sound by the Atmosphere".

${ }^{18}$ Ahuja, K. K. and Tester, B. J., "The Free Jet as a Simulator of Forward Velocity Effects on Jet Noise," NASA CR 3056 , 1978.

${ }^{19}$ Gillian, R. E., Brown, C. G., Bartlett, R. W., and Baucom, P. H., “ANOPP Programmers' Reference Manual for the Executive System," NASA TM X-74029, 1977. 